\title{
Activated protein C therapy slows ALS-like disease in mice by transcriptionally inhibiting SOD1 in motor neurons and microglia cells
}

\author{
Zhihui Zhong, ${ }^{1}$ Hristelina Ilieva, ${ }^{2}$ Lee Hallagan, ${ }^{1}$ Robert Bell, ${ }^{1}$ Itender Singh, ${ }^{1}$ Nicole Paquette, ${ }^{1}$ \\ Meenakshisundaram Thiyagarajan, ${ }^{1}$ Rashid Deane, ${ }^{1}$ Jose A. Fernandez, ${ }^{3}$ Steven Lane, ${ }^{1}$ \\ Anna B. Zlokovic, ${ }^{1}$ Todd Liu, ${ }^{1}$ John H. Griffin, ${ }^{3}$ Nienwen Chow, ${ }^{4}$ Francis J. Castellino, ${ }^{5}$ \\ Konstantin Stojanovic, ${ }^{1}$ Don W. Cleveland, ${ }^{2}$ and Berislav V. Zlokovic ${ }^{1}$
}

\begin{abstract}
${ }^{1}$ Center for Neurodegenerative and Vascular Brain Disorders and Department of Neurological Surgery, University of Rochester Medical Center, Rochester, New York, USA. ${ }^{2}$ Ludwig Institute for Cancer Research, Department of Medicine, and Department of Neuroscience, University of California, San Diego, La Jolla, California, USA. ${ }^{3}$ Department of Molecular and Experimental Medicine, The Scripps Research Institute, La Jolla, California, USA. ${ }^{4}$ Socratech Research Laboratories, Rochester, New York, USA. ${ }^{5}$ WM Keck Center for Transgene Research, Department of Chemistry and Biochemistry, University of Notre Dame, Notre Dame, Indiana, USA.
\end{abstract}

\begin{abstract}
Activated protein C (APC) is a signaling protease with anticoagulant activity. Here, we have used mice expressing a mutation in superoxide dismutase-1 (SOD1) that is linked to amyotrophic lateral sclerosis (ALS) to show that administration of APC or APC analogs with reduced anticoagulant activity after disease onset slows disease progression and extends survival. A proteolytically inactive form of APC with reduced anticoagulant activity provided no benefit. APC crossed the blood-spinal cord barrier in mice via endothelial protein C receptor. When administered after disease onset, APC eliminated leakage of hemoglobin-derived products across the blood-spinal cord barrier and delayed microglial activation. In microvessels, motor neurons, and microglial cells from SOD1-mutant mice and in cultured neuronal cells, APC transcriptionally downregulated SOD1. Inhibition of SOD1 synthesis in neuronal cells by APC required protease-activated receptor-1 (PAR1) and PAR3, which inhibited nuclear transport of the Sp1 transcription factor. Diminished mutant SOD1 synthesis by selective gene excision within endothelial cells did not alter disease progression, which suggests that diminished mutant SOD1 synthesis in other cells, including motor neurons and microglia, caused the APCmediated slowing of disease. The delayed disease progression in mice after APC administration suggests that this approach may be of benefit to patients with familial, and possibly sporadic, ALS.
\end{abstract}

\section{Introduction}

Activated protein C (APC) is an endogenous plasma protease with anticoagulant activity and direct cytoprotective activities (1). The anticoagulant action of APC is mediated by irreversible proteolytic inactivation of factors Va and VIIIa in plasma with contributions of different cofactors. Independent of its anticoagulant action, the protein $\mathrm{C}$ cellular pathway mediates cytoprotective alterations in gene expression (2-4) and controls activation of several transcription factors that regulate different antiapoptotic and antiinflammatory pathways $(2,4-6)$. Most studies have indicated that protease activated receptor-1 (PAR1) is a key receptor mediating APC's transmembrane signaling in different cell types (1). APC protects neurons (7) and endothelial cells (8-10) from different types of injury and limits brain damage in rodent models of ischemia $(6,11-13)$ and multiple sclerosis (14). Whether APC can influence a chronic neurodegenerative process like that in amyotrophic lateral sclerosis (ALS) is unknown.

Mutations in SOD1 are the most-studied forms of inherited ALS (15). Neurodegeneration in SOD1 mutants is mediated via

Authorship note: Zhihui Zhong and Hristelina Ilieva contributed equally to this work.

Conflict of interest: B.V. Zlokovic is a scientific founder of ZZ Biotech LLC, a startup biotech company with a mission to develop new treatments for the aging brain, stroke, and Alzheimer disease. B.V. Zlokovic and J.H. Griffin are inventors on issued and pending patents related to APC.

Citation for this article: J. Clin. Invest. 119:3437-3449 (2009). doi:10.1172/JCI38476 mechanism(s) involving mutant damage within both motor neurons and non-neuronal cells (15) such as microglia (16) and astrocytes (17), both of which develop mutant-mediated damage that drives rapid disease progression. In addition, microvessels control integrity of the blood-spinal cord barrier (BSCB) and are damaged early in the disease process $(18,19)$, allowing leakage of potentially neurotoxic blood components into the spinal cord (19). Using WT recombinant APC, APC variants whose anticoagulant activity is reduced (3K3A-APC; ref. 20) or minimal (5A-APC; refs. 21, 22), or a mutant that has minimal anticoagulant activity but is proteolytically inactive (S360A-APC), we tested whether peripherally administered APC slows the course of motor neuron disease in transgenic mice expressing ALS-linked mutant human superoxide dismutase-1 (SOD ${ }^{\mathrm{G} 93 \mathrm{~A}}$; ref. 23 ). We found that APC with protease activity initially crosses the BSCB via endothelial protein $\mathrm{C}$ receptor (EPCR) and acts on motor neurons and their glial neighbors, especially microglia, to directly inhibit disease progression by reducing mutant SOD1 transcription.

\section{Results}

APC treatment delivered after disease onset controls progression of ALS-like disease. We randomly assigned 60 male mice expressing ALS-linked mutant SOD1 ${ }^{\mathrm{G} 93 \mathrm{~A}}$ into 5 groups receiving saline or each of 4 different recombinant murine APC analogs: (a) WT-APC; (b) 3K3A-APC, which contains 3 alanine substitutions for 3 protease domain residues (Lys191-193) and reduces factor Va binding and inactivation 
but does not affect those APC exosites recognizing PAR1 and EPCR, resulting in greatly reduced anticoagulant activity $(>70 \%$ reduction; ref. 24) and normal cytoprotective activity (20); (c) 5A-APC, which contains 5 alanine substitutions for 5 protease domain positively charged residues (Arg229/230 and Lys191-193) and alters factor Va binding exosites, but not exosites that recognize PAR1 and EPCR, resulting in approximately $10 \%$ of the anticoagulant activity of WTAPC but retaining normal cytoprotective activity $(21,22)$; and (d) S360A-APC, an enzymatically inactive form of APC that lacks serine protease activity and the ability to activate PAR1 (5).

APC treatments or saline were delivered approximately 7 days after disease onset, as determined by denervation-mediated muscle atrophy and accompanying weight loss $(16,25-27)$, which began at about $77 \pm 5.1$ days (Figure 1A). Treatments continued daily throughout the symptomatic phase until death. WT-APC and 3K3A-APC were given at a low dose of $40 \mu \mathrm{g} / \mathrm{kg} / \mathrm{d}$ i.p. With this regimen, plasma APC levels were elevated over a 3 -hour period, with a peak that was about 2.3-fold higher than basal mouse endogenous plasma APC levels (Figure 1B). The half-life of injected APC was 25 minutes. At the higher dose of $100 \mu \mathrm{g} / \mathrm{kg} / \mathrm{d}$ i.p., 5A-APC, inactive S360A-APC, and WT-APC caused 5.8-fold higher plasma APC peak elevations (0.8-0.9 $\mathrm{nmol} / \mathrm{l}$ ) within 15-20 minutes of administration (Figure 1B).

Injection of enzymatically inactive S360A-APC at $100 \mu \mathrm{g} / \mathrm{kg} / \mathrm{d}$ i.p. did not have any significant effect on survival, lifespan, or duration of the symptomatic phase (Figure 1, C-E), whereas enzymatically active WT-APC and 3K3A-APC (both $40 \mu \mathrm{g} / \mathrm{kg} / \mathrm{d}$ i.p.) yielded comparable increases in lifespan (by $10 \%$ and $13 \%, P<0.05$ to $P<0.01$ ) and disease duration (by $23 \%$ and $28 \%, P<0.05$ ) compared with saline-treated controls (Figure 1, D and E). At this initial low dose, the effects of 3K3A-APC on lifespan and duration of the symptomatic phase were not significantly different from those of WT-APC.

Treatment with enzymatically active $5 \mathrm{~A}-\mathrm{APC}$ at $100 \mu \mathrm{g} / \mathrm{kg} / \mathrm{d}$ i.p., administered 1 week after disease onset, increased lifespan from $122 \pm 4$ to $150 \pm 12$ days (a $25 \%$ extension; $P<0.001$ ) and the symptomatic phase from $44 \pm 4$ to $72 \pm 12$ days (64\% longer; $P<0.05$ ) compared with saline-treated mice (Figure 1, C-E). Clotting time of both the ineffective SA360A-APC and the diseaseslowing 5A-APC was reduced similarly (Figure 1G), comparable to values previously reported for human S360A-APC (28), whereas 5A-APC retained fully amidolytic activity and S360A-APC was inactive (Figure 1F). Thus, the serine protease activity of APC, but not its anticoagulant activity, is critical for the observed beneficial effects in slowing disease in SOD1 mutant-mediated ALS, which suggests that PARs are likely involved (1).

APC crosses the blood-spinal cord barrier via EPCR. APC, 3K3A-APC, and $5 \mathrm{~A}-\mathrm{APC}$ cross the blood-brain barrier (BBB) in different brain regions via EPCR-dependent receptor-mediated transport (29). To determine whether slowing disease progression in SOD $1^{\mathrm{G} 93 \mathrm{~A}}$ mice correlates with 5A-APC action within the spinal cord, ${ }^{125}$ I-radiolabeled 5A-APC (125I-5A-APC) was used to determine the concentrations of $5 \mathrm{~A}-\mathrm{APC}$ in the spinal cord interstitial fluid (ISF) in nontransgenic and SOD $1^{\mathrm{G} 93 \mathrm{~A}}$ mice treated with $5 \mathrm{~A}-\mathrm{APC}$ or saline. ${ }^{125} \mathrm{I}-5 \mathrm{~A}-\mathrm{APC}$ levels in the lumbar ISF - determined 15 minutes after i.p. injection of $100 \mu \mathrm{g} / \mathrm{kg}^{125} \mathrm{I}-5 \mathrm{~A}-\mathrm{APC}$, a time point within the initial linear phase of ${ }^{125} \mathrm{I}-5 \mathrm{~A}-\mathrm{APC}$ accumulation in the arterial plasma (Figure 2A) - was identical to that of unlabeled 5A-APC (Figure 1B). After correcting for the residual vascular radioactivity (see Methods), substantial 5A-APC accumulation (to about $3 \mathrm{nmol} / \mathrm{l}$ ) was observed in the spinal cord ISF in normal mice (Figure 2B). 5A-APC uptake was reduced by greater than $80 \%$ in severely depleted EPCR mice
(Figure 2C), but not in PAR1-null mice (Figure 2B), consistent with a requirement for EPCR for APC transport across the BSCB. There was no change in entry of ${ }^{99 \mathrm{~m}} \mathrm{Tc}$-albumin into the spinal cord in comparing EPCR-depleted and control mice (data not shown).

$5 \mathrm{~A}-\mathrm{APC}$ accumulation in the lumbar ISF doubled in naive SOD $1^{\mathrm{G} 93 \mathrm{~A}}$ mice compared with nontransgenic mice (Figure 2D), as expected from the reported BSCB disruption in SOD1G93A mutants $(18,19)$. Increased 5A-APC entry was not caused by an increase in EPCR (Figure 2, E and F), but was eliminated after treatment of comparably aged SOD $1{ }^{\mathrm{G} 93 \mathrm{~A}}$ mice with 5A-APC (Figure $2 \mathrm{D}$ ), consistent with the observed stabilization of the BSCB permeability in response to APC therapy (see below).

APC lowers SOD1 expression in motor neurons of SOD1 $1^{\mathrm{G} 93 A}$ mice. APC modulates expression of several genes, including nuclearly encoded mitochondrial SOD2 (2-4), and suppresses activity of transcription factors such as NF-KB $(2,6)$ and p53 $(4,5)$. To examine whether APC therapy affects SOD1 expression in motor neurons in vivo, mRNA levels were determined in laser-captured spinal cord motor neurons from treated and untreated SOD $1^{\mathrm{G} 93 \mathrm{~A}}$ mice 4 weeks after disease onset. Levels of transgene encoded SOD $1^{\mathrm{G} 93 \mathrm{~A}}$ mRNA (transcribed from the authentic human SOD1 promoter), as well as levels of endogenous mouse SOD1 mRNA, were approximately 40\% lower in motor neurons isolated from 5A-APC-treated mice compared with saline-treated controls (Figure 3A). Treatment with S360AAPC did not affect SOD1 expression. Immunoblotting of extracts of cell populations enriched in motor neurons (greater than $85 \%$ motor neurons) from lumbar spinal cords of 5A-APC-treated and control mice confirmed a significant $50 \%$ reduction in SOD $1^{\mathrm{G} 93 \mathrm{~A}}$ and endogenous mouse SOD1 protein levels $(P<0.05$; Figure 3 , $\mathrm{B}$ and $\mathrm{C})$. Treatment of differentiated N2a neuroblastoma cells expressing an ALS-linked mutant SOD1 ${ }^{\mathrm{G} 85 \mathrm{R}}$ (N2a-SOD1 ${ }^{\mathrm{G} 85 \mathrm{R}}$ cells) confirmed that APC can act directly on neuronal cells to selectively downregulate SOD $1^{\mathrm{G} 85 \mathrm{R}}$ and endogenous mouse SOD1 mRNA (Figure 4A) and protein levels (Figure 4, B and C) to about 40\%-60\% their initial levels. Similar $\mathrm{EC}_{50}$ values were found for reduction in SOD1 mRNA levels by WT-APC and 5A-APC (0.56 \pm 0.09 and $0.51 \pm 0.09 \mathrm{nM}$, respectively; Figure 4D), consistent with their comparable cytoprotective effects in endothelial cells (21).

Neuroprotection by APC correlates with PAR1- and PAR3-dependent decrease in SOD1. PAR1 is a principal signaling receptor for APC in different cell types $(1,3-7,13)$, and PAR3 is apparently required for APC action on neuronal cells $(7,30)$. As in non-neuronal cells (31), pulldown experiments confirmed that PAR1 and PAR3 coexist in neuronal cells, at least in part, as heterodimers (Supplemental Figure 1; supplemental material available online with this article; doi:10.1172/JCI38476DS1). Antibody inhibition was used to determine whether downregulation of mutant SOD1 provided by APC was mediated through any of the 4 PAR receptors, all of which were determined by immunoblotting to be expressed in differentiated neuroblastoma N2a cells (see Supplemental Methods for PAR-specific blocking antibodies). Blockage of PAR1 and PAR 3 activation, but not that of PAR 2 and PAR4, abolished 5A-APC-mediated downregulation of SOD1 (Figure 4E), similar to what has previously been reported in murine cortical neurons $(7,30)$. WT-APC and 5A-APC protected N2a-SOD1 ${ }^{\mathrm{G} 85 \mathrm{R}}$ cells from xanthine/xanthine oxidase-induced stress in a dose-dependent manner (Figure $4 \mathrm{G}$ ), with comparable $\mathrm{EC}_{50}$ values of $0.70 \pm 0.13$ $\mathrm{nM}$ and $0.63 \pm 0.17 \mathrm{nM}$, respectively. APC-mediated protection was again dependent on PAR1 and PAR3 levels, as it was blocked by antibody addition (Figure 4F) and by small interfering RNA 
A

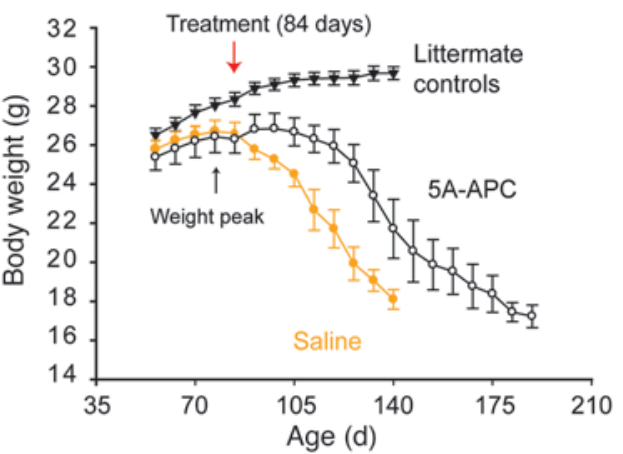

C

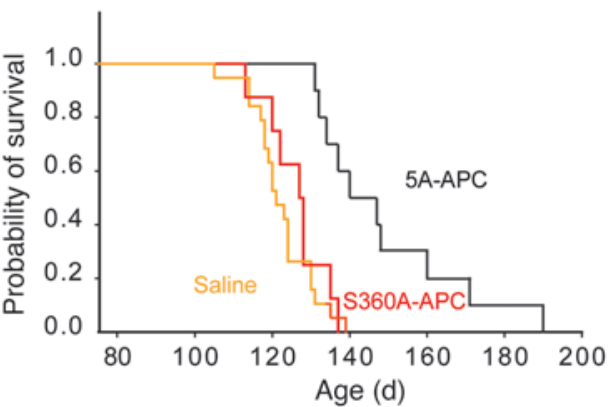

E
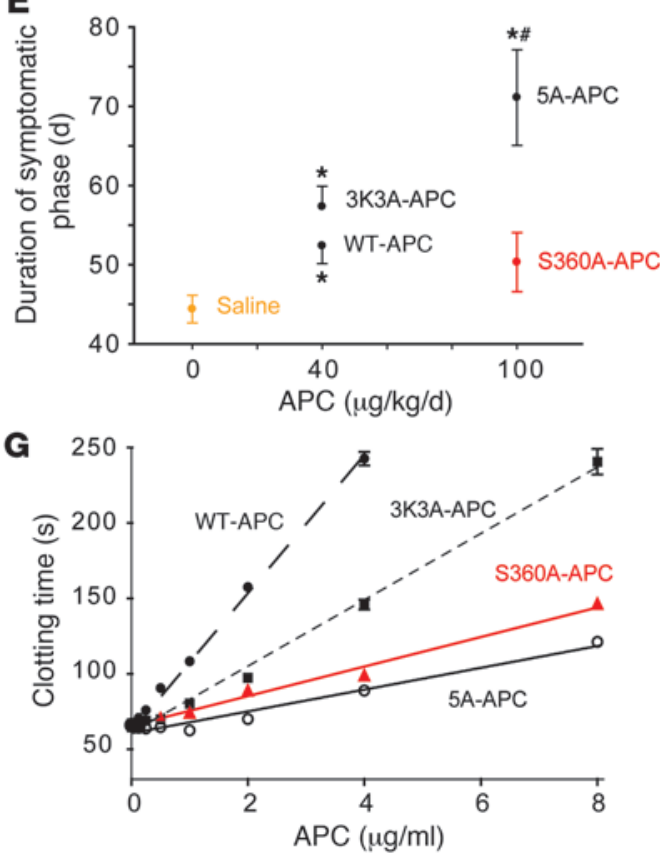

B

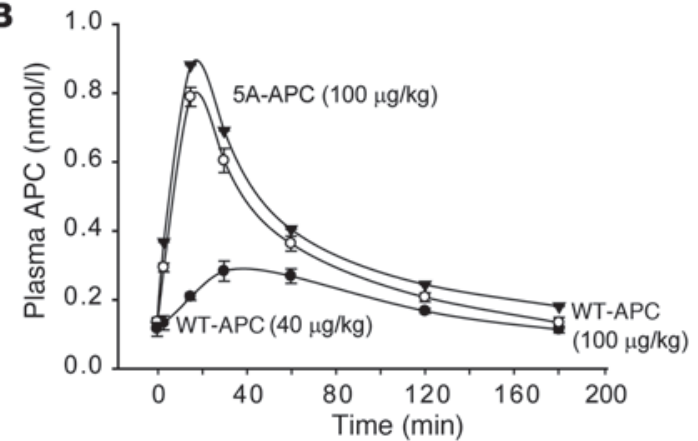

D

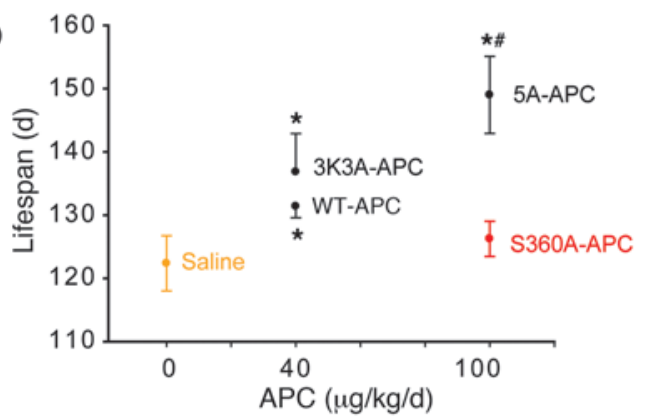

F

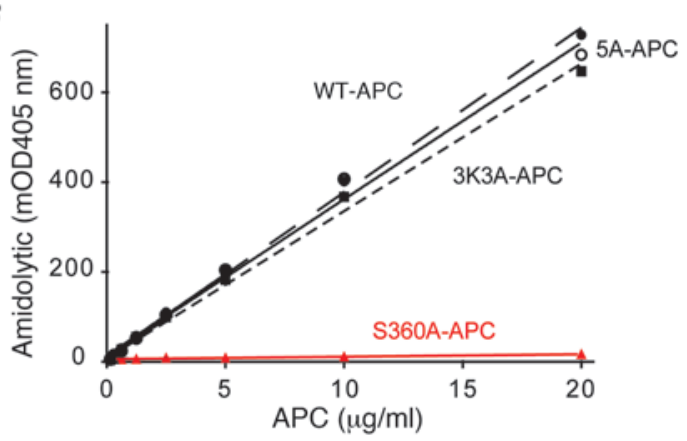

\section{Figure 1}

APC analogs delivered after disease onset control disease progression in SOD1 ${ }^{\text {G93A }}$ mice. (A) Weight curves of SOD1 ${ }^{\text {G93A }}$ mice treated with saline $(n=19)$ or 5A-APC (100 $\mu \mathrm{g} / \mathrm{kg} / \mathrm{d}$ i.p.; $n=10)$ after disease onset (84 days; red arrow) and in nontransgenic littermate controls $(n=12)$. ${ }^{*} P<0.05,5$ A-APC versus saline treatment, repeated-measures ANOVA. (B) APC arterial plasma profiles after i.p. administration of WT-APC $(40 \mu \mathrm{g} / \mathrm{kg} / \mathrm{d}$, filled circles; $100 \mu \mathrm{g} / \mathrm{kg} / \mathrm{d}$, open circles) or 5 A-APC $(100 \mu \mathrm{g} / \mathrm{kg} / \mathrm{d}$, triangles $)$ determined by ELISA. $n=3$. (C) Cumulative probability of survival in SOD1G93A mice treated with saline $(n=19)$, S360A-APC $(100 \mu \mathrm{g} / \mathrm{kg} / \mathrm{d}$ i.p.; $n=10)$ or 5 A-APC $(100 \mu \mathrm{g} / \mathrm{kg} / \mathrm{d} ; n=10)$. (D and E) Lifespan (D) and duration of symptomatic phase (E) in SOD1G93A mice treated i.p. with saline $(n=19)$, WT-APC $(40 \mu \mathrm{g} / \mathrm{kg} / \mathrm{d} ; n=10)$, 3K3A-APC $(40 \mu \mathrm{g} / \mathrm{kg} / \mathrm{d} ; n=11)$, or S360A-APC or 5A-APC (as in C). Differences were calculated from the survival curves by the Cox proportional hazard method (D) and by 1 -way ANOVA followed by Tukey post-hoc test $(\mathrm{E})$. ${ }^{*} P<0.05$ versus saline; ${ }^{*} P<0.05$ versus S360A-APC. S360A-APC was not significantly different from saline. (F and $\mathbf{G}$ ) Amidolytic activity of $(\mathbf{F})$ and clotting time for $(\mathbf{G})$ WT-APC (filled circles), 3K3A-APC (filled squares), 5A-APC (open circles), and S360A-APC (triangles). 

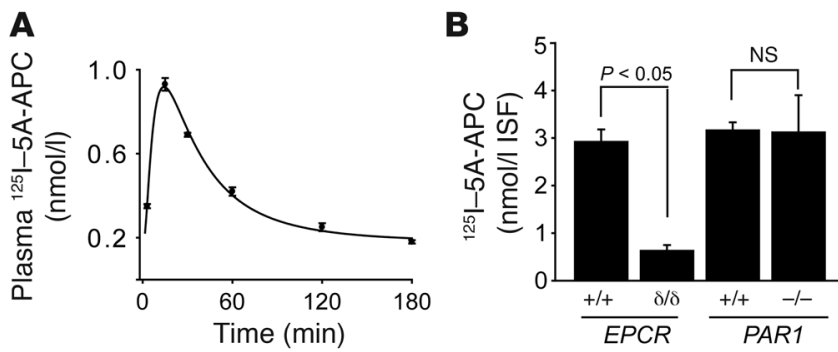

C

D

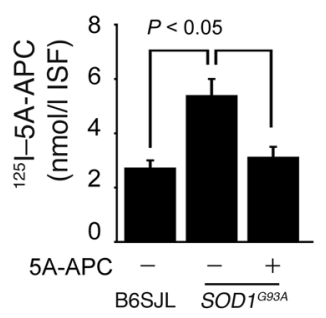

E

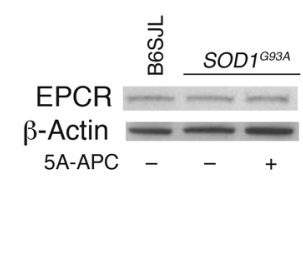

Figure 2

Uptake of radiolabeled 5A-APC by the spinal cord. (A) Arterial plasma profile of ${ }^{125}$ I-5A-APC (TCA-precipitable ${ }^{125} /$-radioactivity) after i.p. injection at $100 \mu \mathrm{g} / \mathrm{kg} . n=3$. (B) Transport of circulating ${ }^{125} \mid-5 A-A P C$ into the lumbar cord ISF in severely depleted EPCR mice $\left(E P C R^{\delta / \delta}\right), P A R 1^{-/-}$mice, and their matching littermate controls (all on C57BL/6 background) after i.p. injection of $125 /-5 A-A P C ~(100 \mu \mathrm{g} / \mathrm{kg}$ ). Concentration of ${ }^{125}$-5A-APC was calculated from TCA-precipitable ${ }^{125}$-radioactivity corrected for the residual vascular radioactivity (see Methods). $n=3-5$. (C) Immunoblot of EPCR in spinal cord microvessels isolated from severely depleted EPCR mice and littermate controls. (D) Transport of circulating ${ }^{125}$ I-5AAPC into the lumbar cord ISF in nontransgenic B6SJL controls compared with SOD1 ${ }^{\text {G93A }}$ mice treated with 5A-APC $(100 \mu \mathrm{g} / \mathrm{kg} / \mathrm{d})$ or saline for 4 weeks after disease onset. $n=5$. (E) Immunoblot analysis of EPCR in spinal cord microvessels isolated from B6SJL or SOD1 $193 \mathrm{~A}$ mice treated with saline or 5A-APC $(100 \mu \mathrm{g} / \mathrm{kg} / \mathrm{d})$ for 4 weeks after disease onset. $\beta$-Actin was used as a loading control. (F) Densitometry of EPCR signal intensity from experiments in E. $n=3-5$.

reduction of either receptor, to about $20 \%$ of the initial level. Finally, although another receptor, EPCR, is required for APC effects on endothelial cells (present study and refs. 3, 4, 20, 32, 33), EPCR cannot mediate direct effects of APC on SOD1 levels in cortical neurons, spinal cord motor neurons, or N2a cells: immunoblotting and RT-PCR revealed that these cells did not express it (Supplemental Figure 2 and Figure 2, C and E).

Neuroprotection by APC reduces hemoglobin and ROS toxicity. In SOD1 mutant mice, microhemorrhages of red blood cells release
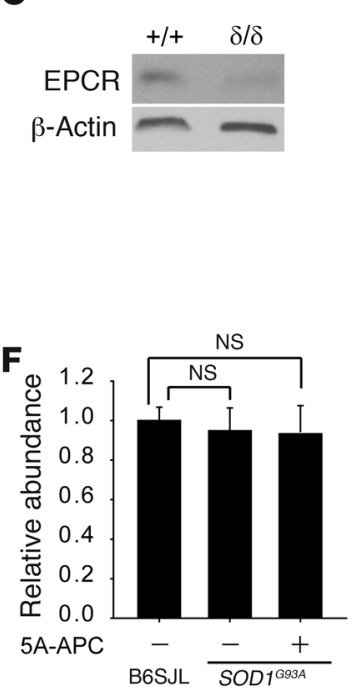

hemoglobin $(\mathrm{Hb})$ and free iron that potentially produce oxidant stress within mutant spinal cords (19). Consistent with this, added $\mathrm{Hb}$ was toxic to N2a cells expressing either dismutaseactive SOD $1^{\mathrm{G} 37 \mathrm{R}}$ or dismutase-inactive SOD1 ${ }^{\text {G85R }}$ mutants (Figure 4H), while similar cells expressing SOD1 ${ }^{\mathrm{WT}}$ were resistant, as previously reported (19). Addition of 5A-APC at concentrations comparable to therapeutically effective levels in SOD1 ${ }^{\mathrm{G} 93 \mathrm{~A}}$ mice (Figure $2 \mathrm{~B}$ ) abolished $\mathrm{Hb}$-induced toxicity (Figure $4 \mathrm{H}$ ) and lowered detectable levels of ROS (Figure 4I). S360A-APC was ineffective in improving cell survival (Figure $4 \mathrm{H}$ ) or preventing ROS generation (data not shown). Reduction in mutant SOD1 by half (through use of shRNA species) blocked Hb toxicity (Figure 4, J and K), just as did the similar mutant SOD1 reduction (Figure 4, A-C) after 5A-APC treatment (Figure $4 \mathrm{H})$. Control shRNA had no effect on SOD $1^{\mathrm{G} 85 \mathrm{R}}$ protein levels and did not protect against $\mathrm{Hb}$ toxicity (Figure 4 , J and K). 5A-APC also protected $\mathrm{N} 2 \mathrm{a}-\mathrm{SOD} 1^{\mathrm{G} 85 \mathrm{R}}$ cells from excitotoxic overstimulation of NMDA glutamate receptors (Figure 4L), consistent with the previously reported action of APC in models of glutamate-induced neuronal excitotoxicity $(7,30,34)$.

The APC-PAR1/PAR3 pathway reduces nuclear levels of Sp1. APC's activation of PAR 1 and PAR3 can suppress activity of transcription factors, including Sp1, Egr-1/WT, NF-אB, and PPAR (35), and/or can activate negative regulators of transcription, such as Yin-Yang 1 or MyoD (36). Because inspection of the human SOD1 transgene construct revealed the absence of NF- $\mathrm{KB}$ and PPAR binding sites (23), and MyoD is not expressed in neuronal cells (37), we tested whether APC affects activation of Sp1, whose DNA-binding activity has previously been shown to be blocked by phosphorylation
A

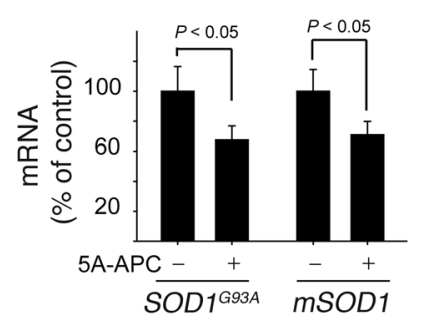

B

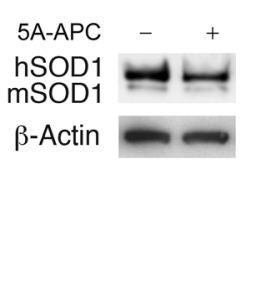

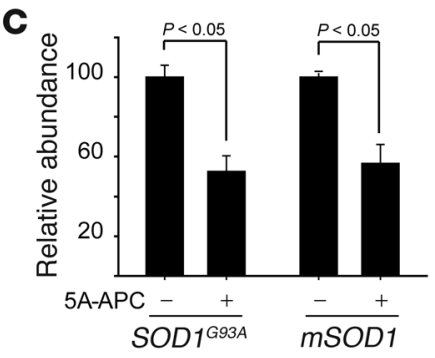

Figure 3

5A-APC transcriptionally downregulates SOD1 expression in spinal cord motor neurons in SOD1G93A mice. (A) Human SOD1G93A and murine mSOD1 mRNA levels were determined by QPCR analysis in laser-captured motor neurons. SOD1G93A mice were treated with 5A-APC (100 $\mu \mathrm{g} / \mathrm{kg} / \mathrm{d}$ i.p.) or saline beginning at 84 days of age, after disease onset. Mice were sacrificed 4 weeks after disease onset. $n=5$. (B) SOD1G93A and mSOD1 protein levels, determined by immunoblot analysis of motor neuron cell lysates from SOD1G93A mice treated as in A. Enriched motor neuron cell populations were isolated from the spinal cord as described in Methods. $n=5$. (C) Scanning densitometry of SOD1G93A and mSOD1 bands in $\mathbf{B}$, relative to $\beta$-actin. $n=3-5$. 
A
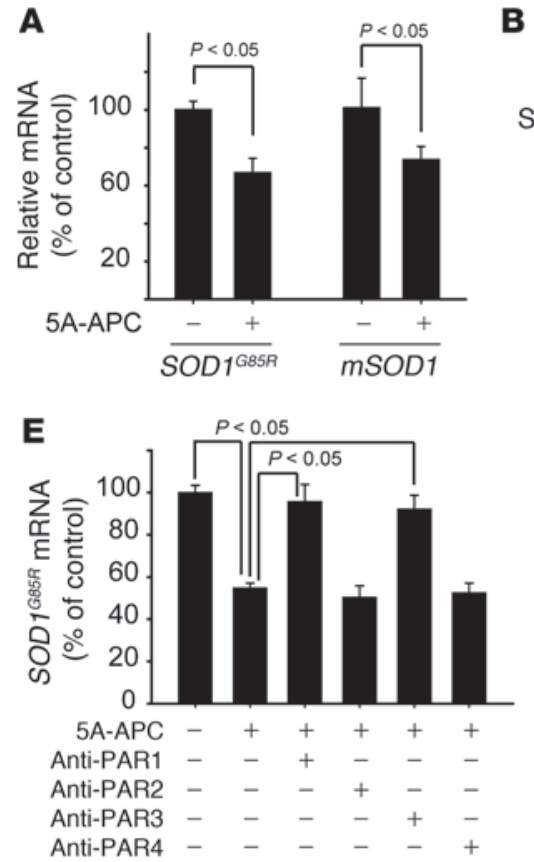

H

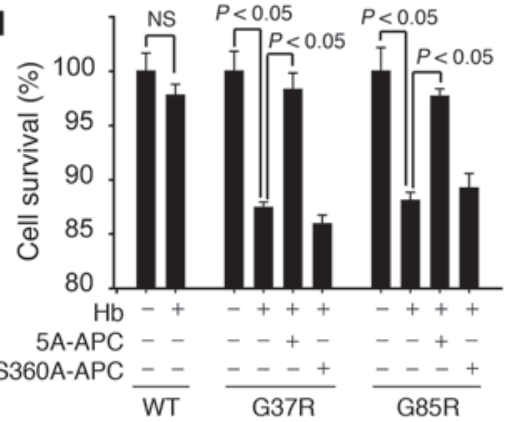

B

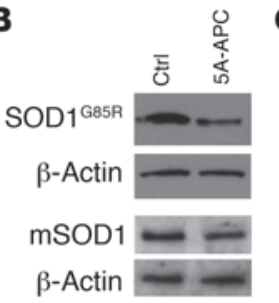

$\mathbf{F}$
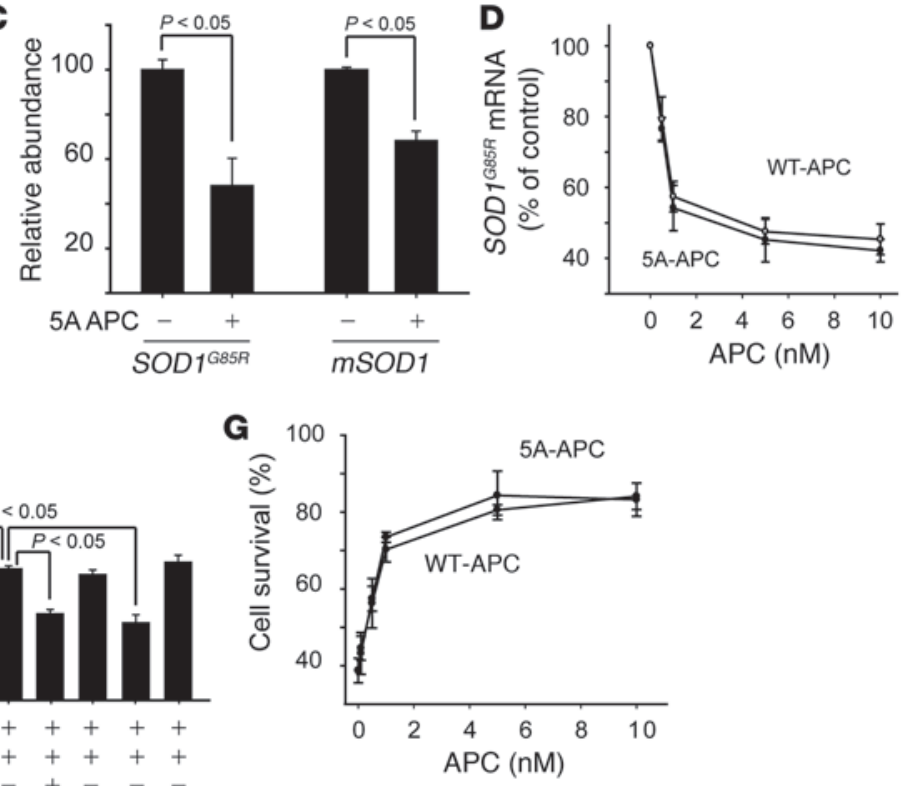

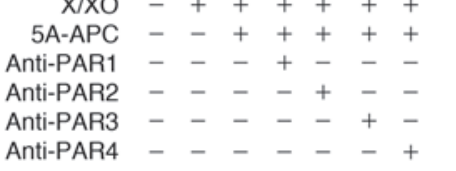

I

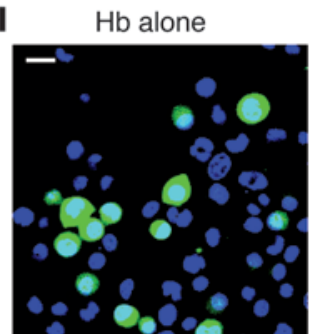

ROS/Hoechst

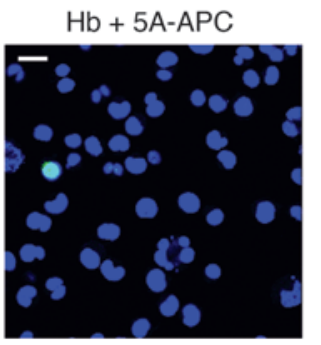

J

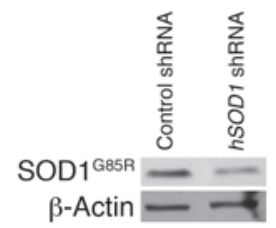

K

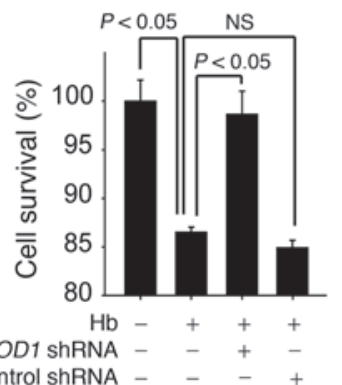

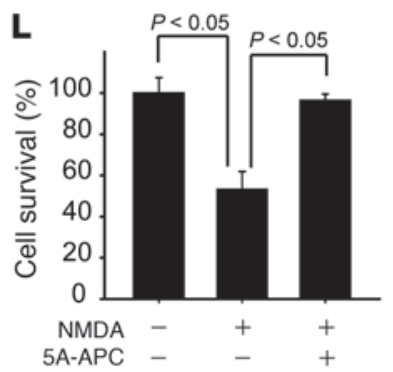

5A-APC

\section{Figure 4}

5A-APC-PAR1/PAR3-mediated SOD1 suppression and neuroprotection in N2a cells. (A) SOD1G93A and mSOD1 mRNA levels, determined by QPCR in N2a-SOD1G85R cells treated with 5A-APC (5 nM) or saline for $48 \mathrm{~h}$. (B) SOD1G93A and mSOD1 protein levels, determined by immunoblot analysis of N2a-SOD1G85R cell lysates in A. (C) Scanning densitometry of SOD1 ${ }^{\mathrm{G} 93 \mathrm{~A}}$ and mSOD1 bands in B, relative to $\beta$-actin. (D and E) SOD1G93A mRNA levels in N2a-SOD1 185R cells treated with 0-10 nM WT-APC or 5A-APC for $48 \mathrm{~h}$ (D) or $5 \mathrm{nM} 5 \mathrm{~A}-\mathrm{APC}$ with and without $20 \mu \mathrm{g} / \mathrm{ml}$ of cleavage site-blocking PAR1, PAR2, PAR3, and PAR4 antibodies (E). (F and G) N2a-SOD1G85R cell viability $16 \mathrm{~h}$ after incubation with xanthine/xanthine oxidase (X/XO) with and without $5 \mathrm{nM} 5 \mathrm{~A}-\mathrm{APC}$ and $20 \mu \mathrm{g} / \mathrm{ml}$ PAR-blocking antibodies (F) and 0-10 nM WT-APC or 5A-APC (G). $(\mathbf{A}-\mathbf{G}) n=5$. (H) Viability of N2a-SOD1WT, N2a-SOD1G37R, and N2a-SOD1G85R cells $16 \mathrm{~h}$ after incubation with Hb with and without $5 \mathrm{nM} 5 \mathrm{~A}-\mathrm{APC}$ or S360A-APC. (I) Double staining for ROS and Hoechst in N2a-SOD1G85R cells $16 \mathrm{~h}$ after incubation with Hb with and without $5 \mathrm{nM} 5 \mathrm{~A}-\mathrm{APC}$ or S360A-APC. Scale bars: $20 \mu \mathrm{m}$. (J) SOD1 reduction in $h$ SOD1 shRNA-transfected N2a-SOD1 G85R cells by immunoblot analysis. (K) Viability of N2a-SOD1G85R cells transfected with hSOD1 shRNA or control shRNA and incubated with Hb as in $\mathbf{H}$. (H-K) $n=3-5$. (L) N2a-SOD1G85R cell viability 24 hours after 10 minutes of exposure to $300 \mu \mathrm{M}$ NMDA with or without $5 \mathrm{nM}$ 5A-APC. $n=5$. 
A
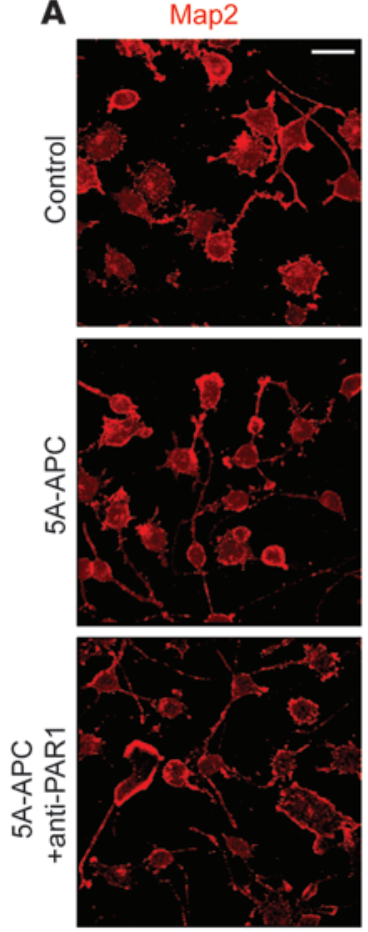

D

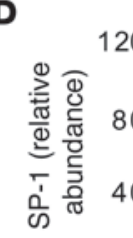

5A-APC anti-PAR1 anti-PAR2 anti-PAR3 anti-PAR4
Sp1
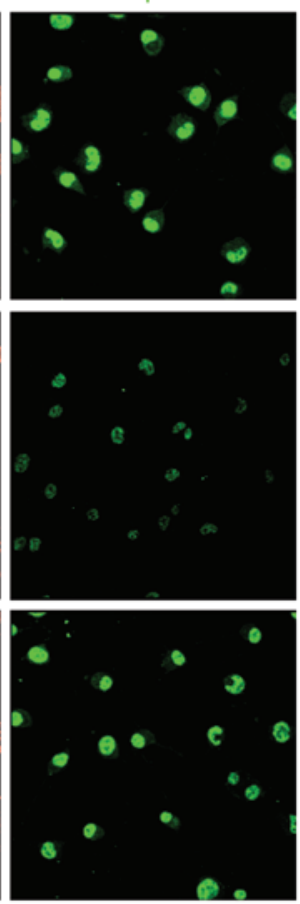

Hoechst
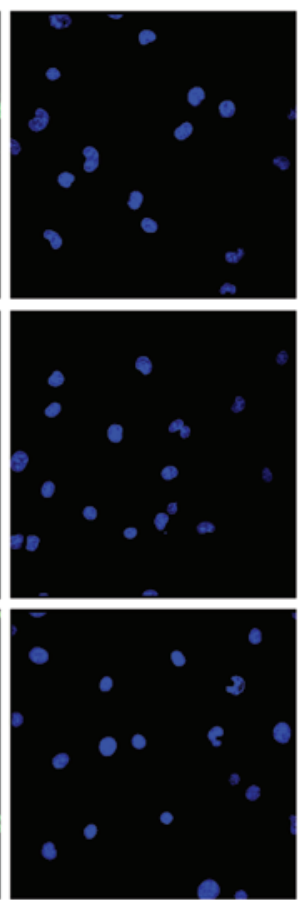

Merged
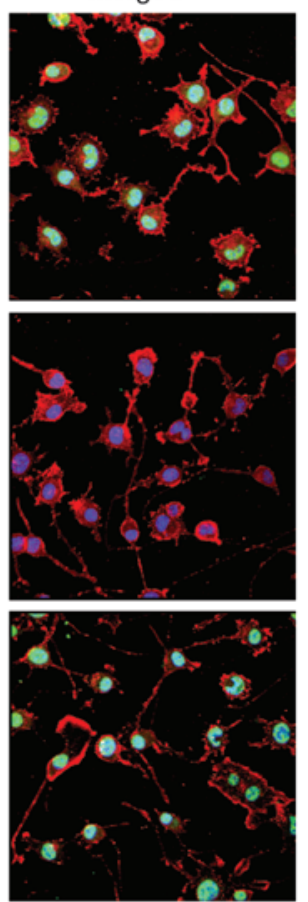

B

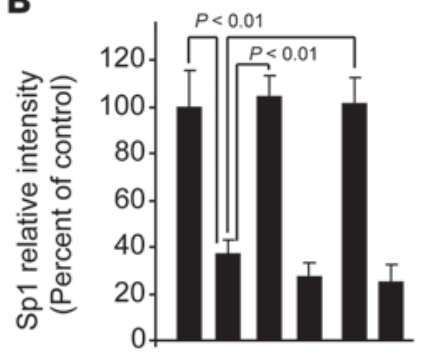

$5 A-A P C$ anti-PAR1 anti-PAR2 anti-PAR3 anti-PAR4

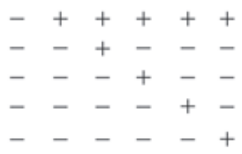

C

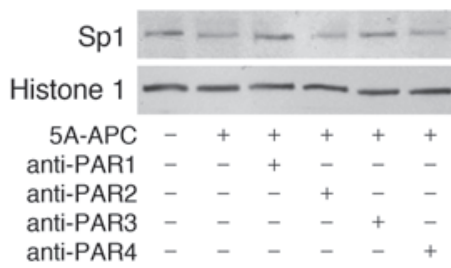

$\mathbf{F}$

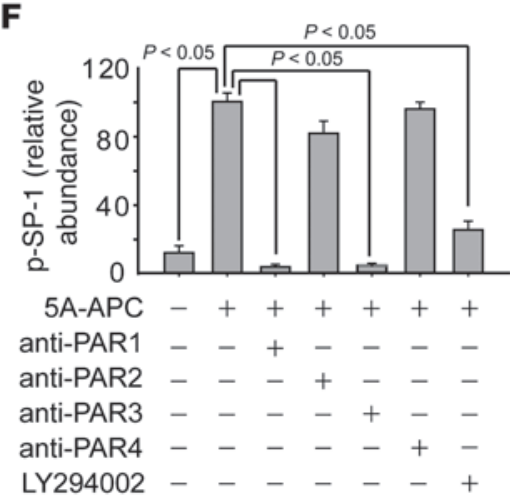

\section{Figure 5}

The 5A-APC-PAR1/PAR3 pathway blocks nuclear translocation of Sp1 in N2a-SOD1G85R_expressing cells. (A and B) Confocal microscopy images (A) and signal intensity (B) of Map2 (red), Sp1 (green), and Hoechst nuclear staining (blue) in N2a-SOD1G85R cells treated with $1 \mathrm{nM}$ 5A-APC for 48 hours with and without PAR1, PAR2, PAR3, and PAR4 cleavage site-blocking antibodies. Scale bar: $20 \mu \mathrm{m}$. $n=5$. (C and D) Immunoblot analysis (C) and densitometry relative to histone 1 (D) of Sp1 in nuclear lysates of N2a-SOD1G85R cells treated as in B. $n=3-5$. (E and F) Immunoblot analysis (E) and scanning densitometry relative to $\beta$-actin (F) of phosphorylated Sp1 in cytoplasmic lysates of N2a-SOD1G85R cells treated as in B or with $50 \mu \mathrm{M}$ of the PI3K inhibitor LY294002. $n=3-5$.

after PAR1 activation (38). We used 3-color confocal microscopy to simultaneously localize Sp1 and the neuronal microtubule-associated protein MAP2 in N2a-SOD1 ${ }^{\mathrm{G} 85 \mathrm{R}}$ cells, and found that almost all of these cells normally expressed nuclear Sp1 (Figure 5A). Treatment with $1 \mathrm{nM} 5 \mathrm{~A}-\mathrm{APC}$ significantly reduced nuclear Sp 1 by $60 \%$ compared with untreated controls $(P<0.01$; Figure 5, A and B). Reductions in nuclear Sp1 were also observed in motor neurons in spinal cords of SOD ${ }^{\text {G93A }}$ mice treated with 5A-APC, but not S360A-APC or saline (data not shown). Antibody inhibition in vitro of PAR1 (Figure 5A) or PAR3, but not PAR2 or PAR4 (Figure 5B), abolished 5A-APC suppression of nuclear Sp1. Quantitative immunoblotting of 5A-APC-treated cells confirmed a $60 \%$ reduc- tion in nuclear $\mathrm{Sp} 1(P<0.05)$ that was blocked by antibodies to PAR1 and PAR3, but not PAR2 and PAR4 (Figure 5, C and D).

Levels of phosphorylated Sp1 in cytoplasmic cell lysates were elevated after 5A-APC treatment of N2a-SOD1 ${ }^{\mathrm{G} 85 \mathrm{R}}$ cells (Figure 5, $\mathrm{E}$ and $\mathrm{F}$ ), consistent with an earlier report suggesting that PAR1 signaling results in SP1 phosphorylation, preventing its nuclear translocation or retention (38). Increased Sp1 phosphorylation was inhibited both by the PI3K inhibitor LY294002 and by PAR1 and PAR3 antibodies. Parallel immunostaining and immunoblotting for 2 other transcription factors whose cis elements are also present in SOD1 transgene, Egr-1 and Yin-Yang, revealed no APC-dependent change in their respective nuclear levels (data not shown). 
A

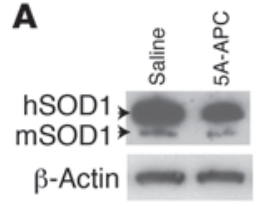

B

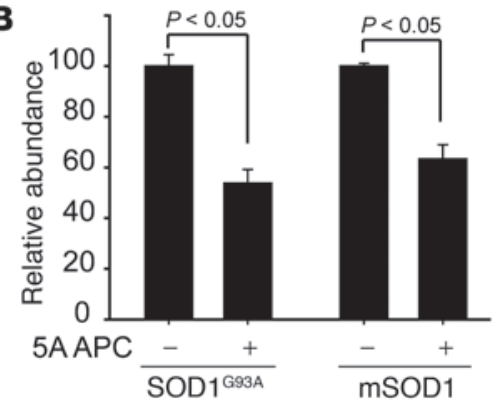

E

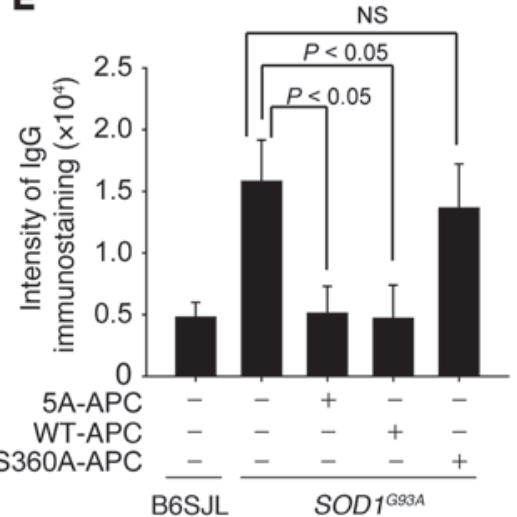

C

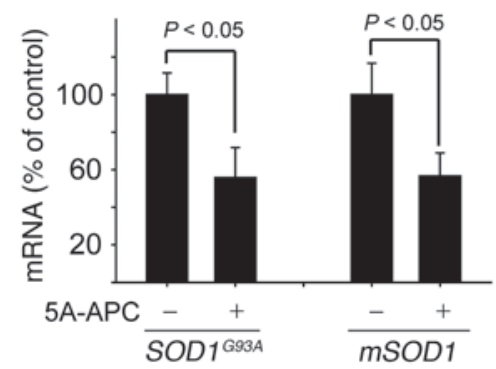

$\mathbf{F}$

F S360A-APC

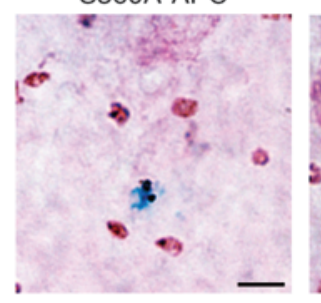

5A-APC

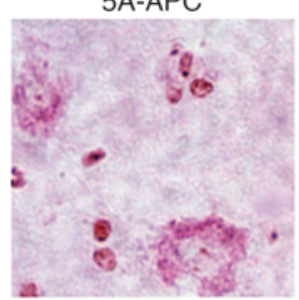

G

NS

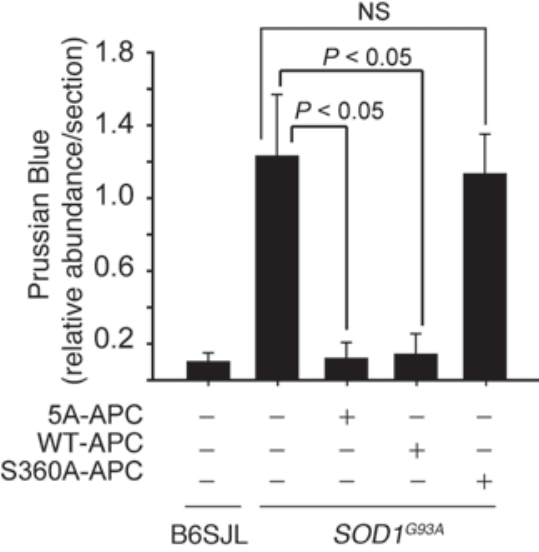

H

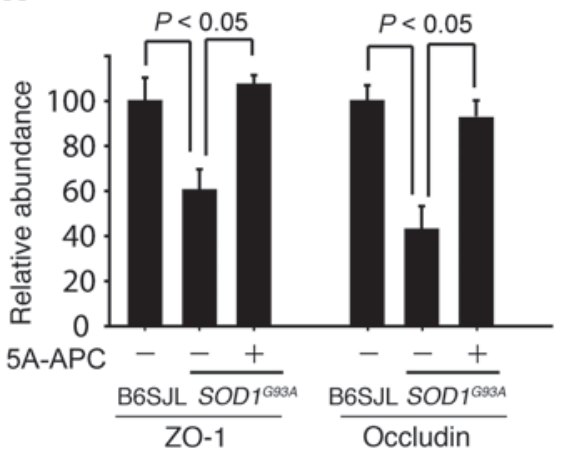

Figure 6

APC downregulates SOD1 in spinal cord microvessels and blocks BSCB disruption in SOD1G93A mice. (A and B) Immunoblotting (A) and densitometry (B) of human SOD1G93A and mouse mSOD1 in spinal cord microvessels isolated from SOD1G93A mice treated with 5A-APC or S360AAPC at $100 \mu \mathrm{g} / \mathrm{kg} / \mathrm{d}$ i.p. for 4 weeks after disease onset. Relative band density was normalized to $\beta$-actin. $n=5$ per group. (C) SOD1G93A and $m S O D 1$ mRNA levels determined by QPCR in laser-captured microvessels from mice as in B. $n=5$ per group. (D) Immunostaining for lgG (green) and endothelium (CD31, red) in the lumbar spinal cords of SOD1G93A mice treated with S360A-APC or 5A-APC as in B. Scale bar: $50 \mu \mathrm{m}$. (E) IgG signal intensity in the lumbar spinal cords of SOD1G93A mice treated as in B or with $40 \mu \mathrm{g} / \mathrm{kg} / \mathrm{d}$ WT-APC. $n=5-8$. (F) Hemosiderin deposits in the lumbar spinal cord of SOD1 ${ }^{\mathrm{G} 93 \mathrm{~A}}$ mice treated as in B. Scale bar: $20 \mu \mathrm{m}$. (G) Quantification of lumbar spinal cord hemosiderin deposits in SOD1G93A mice from $\mathbf{D}$ and $\mathbf{F}$. $n=5-8$. (H) Relative abundance of ZO-1 and occludin, normalized to $\beta$-actin, from immunoblots of spinal cord microvessels isolated from SOD1G93A mice treated with 5A-APC as in $\mathbf{B}$ or with saline. $n=3-5$. (E, G, and $\mathbf{H})$ B6SJL denotes littermate controls.

APC downregulates SOD1 in spinal cord microvessels and maintains the BSCB. A significant $(50 \% ; P<0.01)$ APC-dependent reduction was seen after 4 weeks of APC treatment initiated at disease onset in both SOD $1{ }^{\mathrm{G} 93 \mathrm{~A}}$ and endogenous mouse SOD1 proteins and mRNAs, determined by immunoblotting of extracts from lumbar spinal cord capillaries (Figure 6, A and B) and in the corresponding mRNAs isolated from laser-captured microvessels (Figure 6C). APC treatment after disease onset was also accompanied by greatly reduced damage to the $\mathrm{BSCB}$, as evidenced by blockade of serum protein IgG leakage (Figure 6, D and E) and elimination of microhemorrhages (detected by Prussian blue staining for hemosiderin; Figure 6, F and G). Similarly, a low dose of WT-APC, but not a high dose of S360A-APC, effectively controlled BSCB disruption (Figure $6 \mathrm{E}$ ) and microhemorrhages (Figure 6G). Reduced accumulation of endothelial tight junction proteins ZO-1 and occludin, which function to maintain BSCB integrity (19), was found in SOD1 ${ }^{\mathrm{G} 93 \mathrm{~A}}$ 

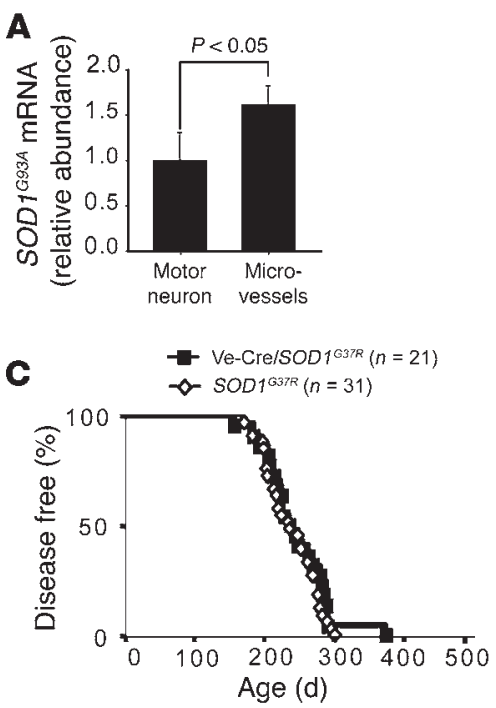

$\mathbf{E}$

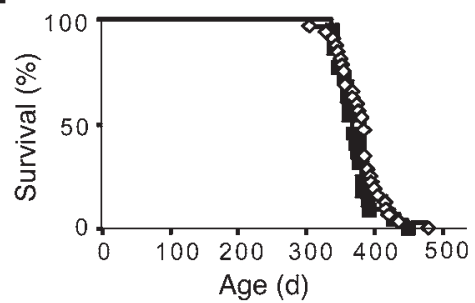

B

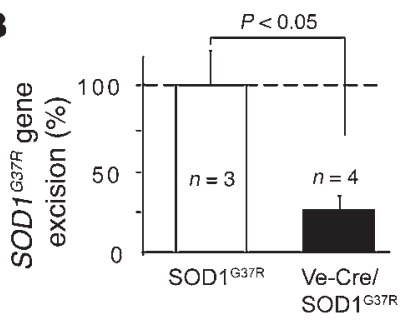

D

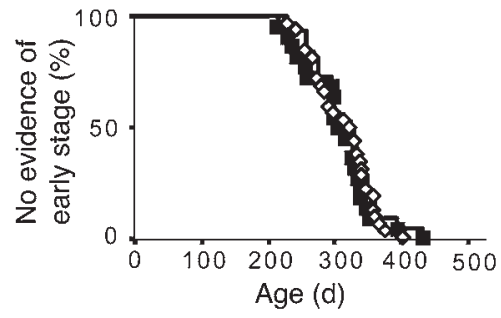

$\mathbf{F}$

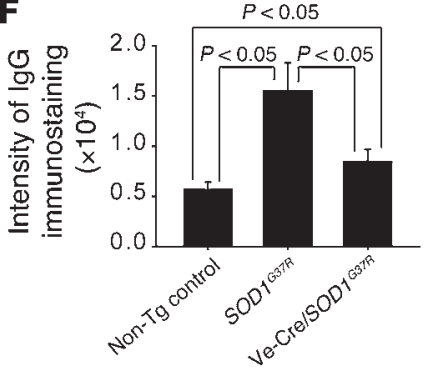

Figure 7

Selective reduction of mutant SOD1 expression within endothelial cells by Cre-mediated gene excision does not affect ALS-like disease onset, progression, or survival. (A) Relative mRNA abundance of SOD1 ${ }^{\mathrm{G} 93 \mathrm{~A}}$ in lasercaptured microvessels and motor neurons in SOD1G93A mice. $n=5$. (B) Excision of the SOD1G37R transgene in microvessels purified from SOD1G37R mice or Ve-Cre/ SOD1G37R mice promoted Cre recombinase transgene. DNA copy number for the mutant SOD1 gene was normalized to mouse apoB gene. From 2-3 mice per extraction, 3 or 4 preparations of vessel-extracted DNA were analyzed. Excision frequency was corrected for the known 4:1 ratio of endothelial cells/pericytes. Data are mean \pm SD. $n=3-4$. (C-E) Age of disease onset (C), progression to an early phenotypic stage (D), and survival (E) in SOD1G37R and Ve-Cre/SOD1G37R mice. (F) IgG signal intensity in the lumbar spinal cords in SOD1G37R mice $(n=4)$ and Ve-Cre/SOD1G37R mice $(n=4)$ at disease onset at 7 months of age compared with agematched nontransgenic littermate controls $(n=3)$. capillaries (Figure 6H and ref. 19). 5A-APC treatment in SOD1G93A mice restored normal ZO-1 and occludin expression (Figure $6 \mathrm{H}$ ).

Selective gene excision of mutant SOD $1^{\mathrm{G} 37 R}$ from endothelial cells does not affect disease. Quantitative RT-PCR (QPCR) was used to confirm that endothelial cells synthesize high levels of SOD1: mutant SOD $1^{\mathrm{G} 93 \mathrm{~A}}$ mRNA levels were $40 \%$ higher in laser-captured spinal cord capillaries than in motor neurons (Figure 7A). Combined with presymptomatic damage to spinal cord capillaries previously observed in SOD1 mutant mice (19), this finding suggested that slowing of disease by APC might be caused by transcriptional reduction of mutant SOD1 within endothelial cells. Prior efforts using cell type-selective expression of the Cre recombinase and a transgenic line carrying a SOD1 mutant gene excisable by Cre action (loxSOD1 ${ }^{\mathrm{G} 37 \mathrm{R}}$ mice) have proven that mutant damage within motor neurons $(16,17)$ drives disease onset, while mutant damage within astrocytes (17) and microglia (16) drives rapid disease progression. As expected, the loxSOD ${ }^{\mathrm{G} 37 \mathrm{R}}$ mice exhibited a leaky BSCB phenotype at disease onset (Figure 7F), consistent with previous findings in other mutant SOD1 mouse lines (19).

To selectively silence mutant SOD1 expression within endothelia, the loxSOD $1^{\mathrm{G} 37 \mathrm{R}}$ mice were mated to a line of mice carrying a Ve-cadherin-promoted Cre transgene previously shown to be selectively expressed in endothelial cells (39), generating Ve-Cre/SOD1 ${ }^{\mathrm{G} 37 \mathrm{R}}$ mice. Gene excision within endothelia was efficient (75\%; Figure 7B) and attenuated BSCB disruption by $70 \%$, relative to comparably aged SOD $1^{\mathrm{G} 37 \mathrm{R}}$ mice, although the Ve-Cre/SOD $1^{\mathrm{G} 37 \mathrm{R}}$ double transgenic mice still had about $50 \%$ greater IgG leakage than did normal mice (Figure 7F). Unlike slowed disease and extended survival from APC treatment, however, highly diminished mutant synthesis within endothelia did not affect the timing of disease onset (Figure 7C), progression to early disease (Figure 7D), survival (Figure 7E), or disease duration (Table 1). Thus, although the timing of BSCB disruption and how different degrees of BSCB disruption affect disease in SOD1 mutants remain to be established, our present results demonstrate that mutant SOD1 synthesized by the capillary endothelia is not an important contributor to pathogenesis and that the benefit in slowing disease progression and extending survival by APC therapy cannot be attributed to mutant SOD1 downregulation in endothelia.

APC downregulates mutant SOD1 in microglia and delays an inflammatory response. Because SOD1 mutant expression within microglia drives rapid disease progression $(16,40)$, we tested whether APC treatment after disease onset affected the levels of SOD1 in microglia or microglial activation. Accompanying 5A-APC treatment was reduction by about $40 \%$ in microglial SOD $1{ }^{\mathrm{G} 93 \mathrm{~A}}$ expression 7 weeks after disease onset (Figure 8A). Administration of low-dose WT-APC or of 5A-APC delayed microglia activation and

\section{Table 1}

Disease onset, early disease, end stage, and duration in SOD1G37R and Ve-Cre/SOD1G37R mice

\begin{tabular}{lcc}
\hline & & \\
& OOD1G37R $_{(\boldsymbol{n}=\mathbf{3 1})}$ & Ve-Cre/SOD1G37R $(\boldsymbol{n}=\mathbf{2 1})$ \\
Age at onset (d) & $240 \pm 37$ & $240 \pm 39$ \\
Age at early disease (d) & $313 \pm 44$ & $301 \pm 47$ \\
Age at end stage (d) & $377 \pm 29$ & $368 \pm 21$ \\
Disease duration (d) & $137 \pm 15$ & $128 \pm 21$ \\
\hline
\end{tabular}

Data are mean \pm SD. 


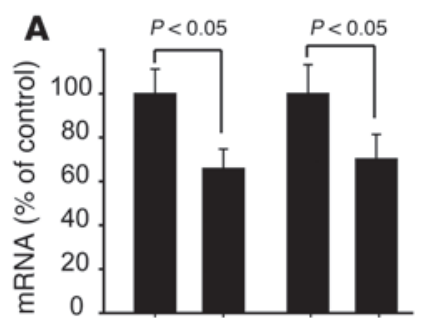

5A-APC $\overline{S O D 1^{G 93 A}} \overline{m S O D 1}$

C

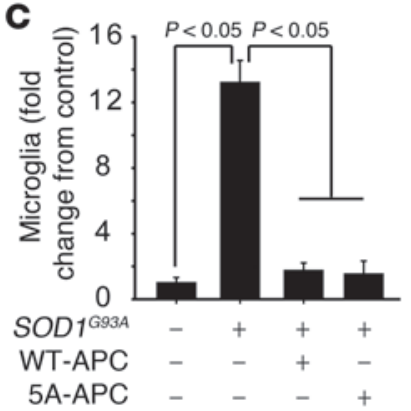

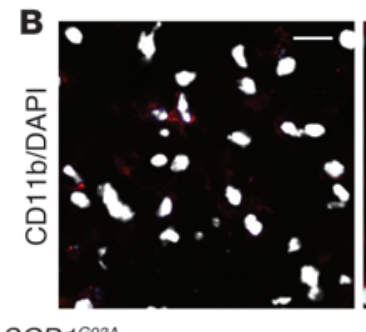

SOD $1^{\text {G93A }}$ WT-APC 5A-APC

D

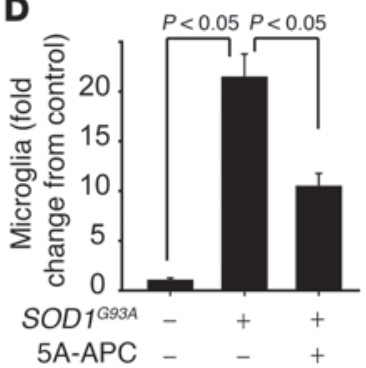

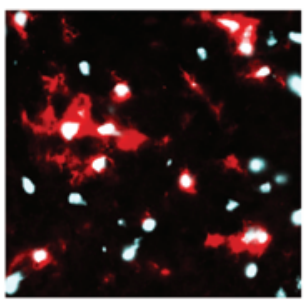

$+$

$-$

E

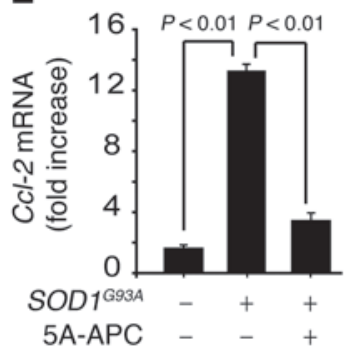

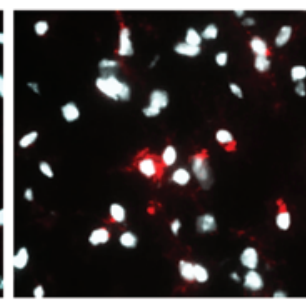

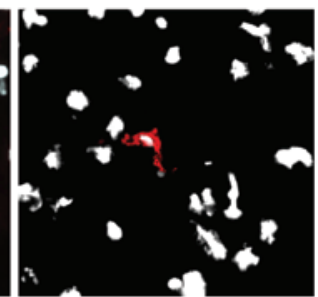

$+$

$+$

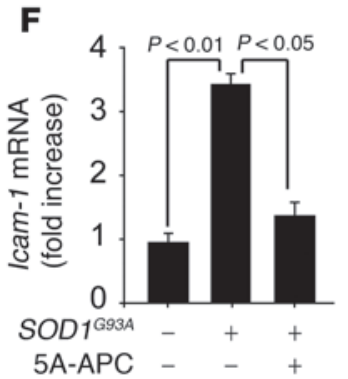

G

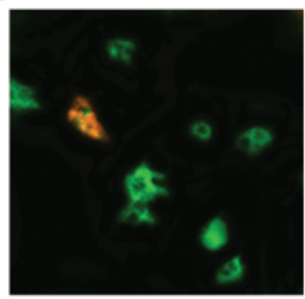

$5 \mathrm{~A}-\mathrm{APC}$

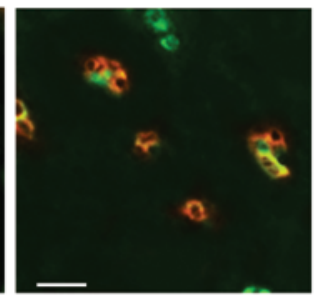

H

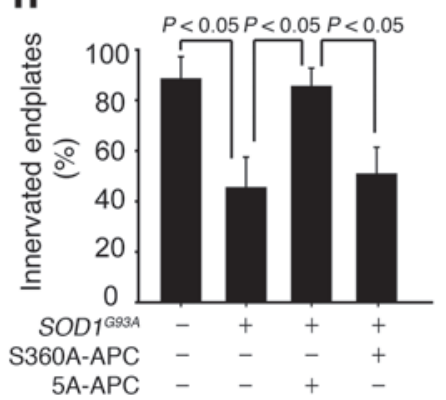

Figure 8

APC downregulates SOD1 in microglia and controls neuroinflammatory response in SOD1G93A mice. (A) QPCR analysis of SOD1G93A and $m S O D 1$ mRNA levels in laser-captured microglia in SOD1G93A mice treated with 5 A-APC or S360A-APC at $100 \mu \mathrm{g} / \mathrm{kg} / \mathrm{d}$ for 7 weeks after disease onset. $n=5$. (B) Immunostaining of activated microglia (CD11b, red) in SOD1 ${ }^{\text {G93A }}$ mice treated with saline, $40 \mu \mathrm{g} / \mathrm{kg} / \mathrm{d} \mathrm{WT-APC}$, or $100 \mu \mathrm{g} / \mathrm{kg} / \mathrm{d} 5 \mathrm{~A}-\mathrm{APC}$ for 4 weeks after disease onset. Nuclei were stained with DAPI (white). Scale bar: $20 \mu \mathrm{m}$. (C) Microglia numbers, in SOD1 ${ }^{\mathrm{G} 93 \mathrm{~A}}$ mice treated as in B, relative to control B6SJL mice (arbitrarily taken as 1). $n=5-6$ per group. (D) CD11b-positive microglia numbers in SOD1G93A mice treated with $100 \mu \mathrm{g} / \mathrm{kg} / \mathrm{d}$ 5A-APC or S360A-APC for 7 weeks. $n=5$ per group. (E and F) QPCR analysis of mRNA transcripts for Ccl-2 (E) and $/ c a m-1$ (F) in the lumbar spinal cords of mice treated with saline or $100 \mu \mathrm{g} / \mathrm{kg} / \mathrm{d} 5 \mathrm{~A}-\mathrm{APC}$ for 4 weeks. $n=3-5$ per group. (G) Double immunostaining of skeletal muscle sections with $\alpha$-bungarotoxin and VAChT, to label endplates and axon terminals, respectively, in SOD $1 \mathrm{G} 93 \mathrm{~A}$ mice treated with $100 \mu \mathrm{g} / \mathrm{kg} / \mathrm{d}$ 5A-APC or S360A-APC for 4 weeks after disease onset. Denervated endplates were labeled only with bungarotoxin (green; left). Innervated endplates were labeled with both bungarotoxin and VAChT (right). Scale bar: $25 \mu \mathrm{m}$. (H) Percentage of innervated plates in $\mathbf{G}$. $n=4$ per group.

proliferation 4 weeks after onset (Figure 8, B-D), while spinal cord levels of microglia in saline-treated SOD ${ }^{\mathrm{G} 93 \mathrm{~A}}$ mice showed increases of 12 - and 20-fold compared with nontransgenic littermate controls at 4 and 7 weeks after onset, respectively (Figure 8, C and D). In contrast to the highly elevated levels in saline-treated SOD $1{ }^{\mathrm{G} 93 \mathrm{~A}}$ mice, as reported previously (19), 5A-APC treatment effectively reduced the expression of inflammatory markers, including monocyte chemoattractant protein 1 (MCP-1, also known as Ccl-2; ref. 40) and Icam-1, a neuroinflammatory marker of activated endothelium (Figure 8, E and F, and ref. 19). 5A-APC treatment also reduced the expression of hemoxygenase- 1 in the spinal cord (Supplemental Figure 3). Moreover, treatment with both WT-APC (data not shown) and 5A-APC (Figure 8, G and H), but not with S360A-APC, substantially preserved innervation of the neuromuscular junctions 4 weeks after onset, an age at which untreated and saline-treated animals had developed significant muscle weakness.

APC-EPCR interactions at the spinal cord endothelium. EPCR ${ }^{\delta / \delta}$ hypomorphs exhibited severely depleted EPCR levels in the spinal cord endothelium (Figure 2C) and were therefore unable to transport WT-APC, 3K3A-APC, or 5A-APC from the circulation across the BBB (29) and into the spinal cord (Figure $2 B$ ). Indeed, in $E P C R^{\delta / \delta}$ hypomorphs, systemic administration of 5A-APC did not reduce SOD1 in motor neurons or spinal cord endothelium in vivo, although, as above, it lowered SOD1 by half in $\mathrm{EPCR}^{+/+}$animals (Figure 9, A-F).

RCR-252, an anti-human EPCR antibody directed to an APC binding site on EPCR (41), has been reported to block murine 
A

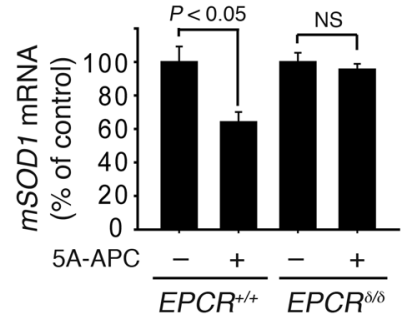

D

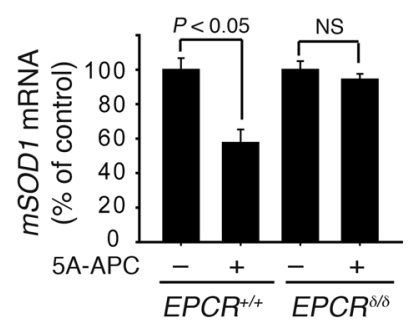

G

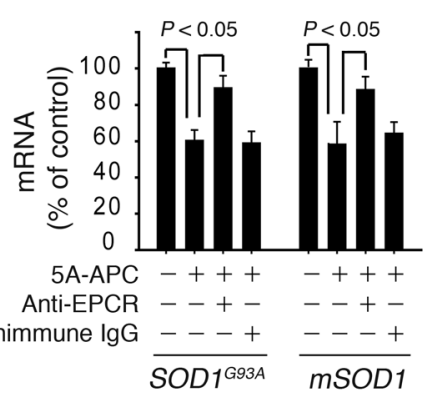

J

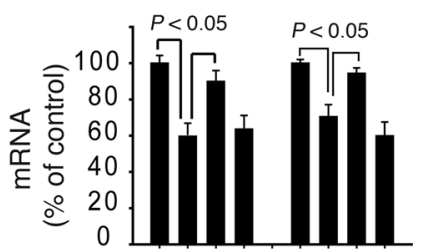

$5 \mathrm{~A}-\mathrm{APC}-++++++$

Anti-EPCR --+---+

Nonimmune $\lg \frac{---+}{S O D 1^{G 93 A}} \frac{--++}{\text { mSOD1 }}$
B

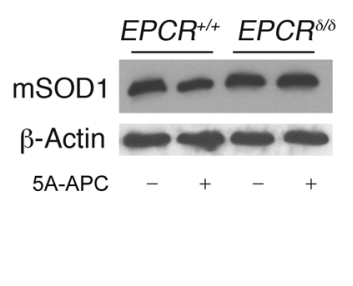

E

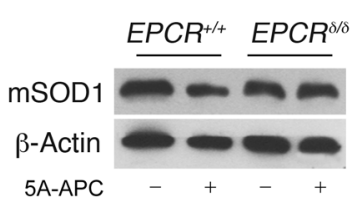

H

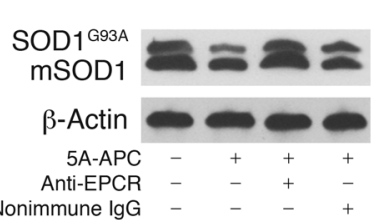

C

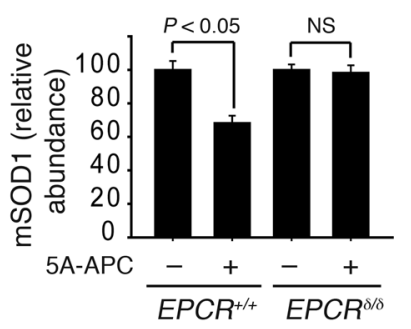

$\mathbf{F}$

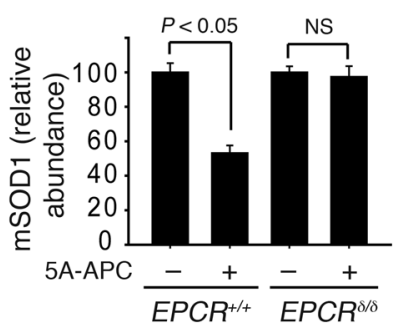

I

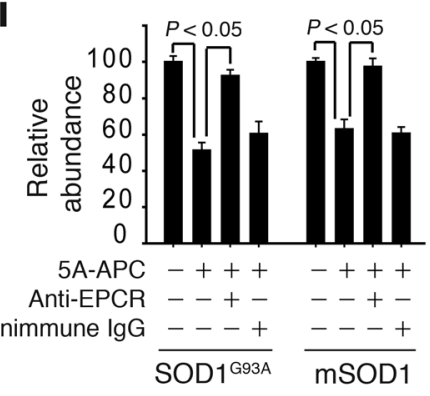

$\mathbf{L}$

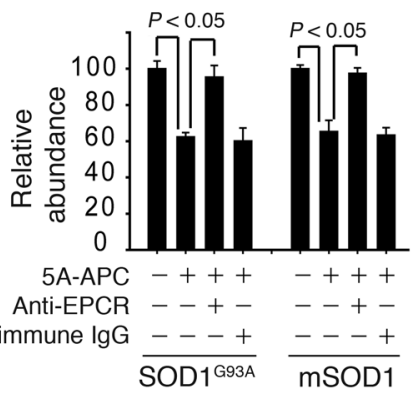

Figure 9

5A-APC-mediated SOD1 downregulation in motor neurons and spinal cord endothelium requires endothelial EPCR. (A-C) mSOD1 mRNA levels, determined by QPCR (A), and immunoblotting (B) and densitometry analysis (C) of mSOD1 protein, in laser-captured motor neurons from $E P C R^{\delta / \delta}$ and $E P C R^{+/+}$mice treated with saline or $100 \mu \mathrm{g} / \mathrm{kg} / \mathrm{d} 5 \mathrm{~A}-\mathrm{APC}$ i.p. for 7 days. (D-F) $m S O D 1 \mathrm{mRNA}$ levels (D), and immunoblotting (E) and densitometry $(\mathbf{F})$ analysis of mSOD1 protein levels, in spinal cord microvessels isolated from $E P C R^{\delta / \delta}$ and $E P C R^{+/+}$mice treated as in $\mathbf{A}-\mathbf{C}$. $(\mathbf{A}-\mathbf{F}) n=3-4$ per group. (G-I) SOD1 ${ }^{\text {G93A }}$ and $m S O D 1$ mRNA levels $(\mathbf{G})$, and immunoblotting (H) and densitometry (I) analysis of SOD1G93A and mSOD1 protein levels, in laser-captured spinal cord motor neurons of SOD1 $\mathrm{G}^{\mathrm{G} 3 \mathrm{~A}}$ mice treated with saline or $100 \mu \mathrm{g} / \mathrm{kg} / \mathrm{d} 5 \mathrm{~A}-\mathrm{APC}$ i.p. for $7 \mathrm{days}$ in the absence or presence of an EPCR blocking antibody (RCR-252) or nonimmune lgG infused through the femoral vein (40 $\mu \mathrm{g} / \mathrm{mouse})$ at day 1 and 3. (J-L) SOD1 ${ }^{G 93 A}$ and $m S O D 1$ mRNA levels (J), and immunoblotting (K) and densitometry (L) analysis of SOD1G93A and mSOD1 protein levels, in spinal cord microvessels isolated from SOD1G93A mice treated as in $\mathbf{G}-\mathbf{I}$. (G-L) $n=3-5$.

EPCR activity in vivo, thereby inhibiting APC transport across the BBB (29). We determined that this antibody also recognized murine EPCR in spinal cord endothelium (Supplemental Figure 2). Systemic administration of this antibody, but not nonimmune IgG used at a comparable concentration, blocked 5A-APC-mediated mutant SOD1 downregulation in SOD ${ }^{\mathrm{G} 93 \mathrm{~A}}$ mice at both the mRNA and the protein levels in motor neurons (Figure 9, G-I) and capillary endothelia (Figure 9, J-L).

\section{Discussion}

We have determined that treatment of mutant SOD1-expressing mice with enzymatically active APC analogs delivered after disease onset retarded progression of ALS-like disease, increased lifespan, and, more importantly, increased duration of the symptomatic phase. The enzymatic activity of APC, but not its anticoagulant activity, was critical for the beneficial effects. A primary mechanism for APC's action is through passage across the BSCB in an EPCR- 
dependent manner, followed by transcriptional downregulation of mutant SOD1 in motor neurons and their non-neuronal neighbors, including microglia and cells comprising microvessels.

BSCB disruption $(18,19)$, swelling $(18,19)$, and ischemic changes $(42)$ are consistent features in spinal cord pathology of ALS mice $(18,19)$. Ischemia worsens motor neuron degeneration and functional outcome, as shown in mice with a mutation that eliminates hypoxia-responsive induction of the vascular endothelial growth factor A gene (42), which develop late-onset motor neuron degeneration (43). Endothelial cells of the microvasculature, along with pericytes and astrocytes, were essential to stabilize the BSCB, but we have also shown that APC's slowing of disease was not mediated by reduction of mutant SOD1 within the endothelial cells, the first cells to encounter plasma-delivered APC. Indeed, mutant SOD1 damage directly within the endothelial cells was shown by selective gene excision to play little, if any, role in disease pathogenesis. Activation of microglia and astrocytes and the accompanying inflammatory response, on the other hand, play a major role in progressive BSCB opening in SOD1 mutants after disease onset $(18,19)$. Thus, we conclude that BSCB stabilization by APCs given postsymptomatically - as in the present study - will critically depend on mutant SOD1 reduction in microglia and astrocytes after APC transport across the capillary wall. Additional transgenic mouse models will be needed to resolve whether the well-described protective effects of APC on endothelium $(8,9,44)$ can improve capillary integrity in SOD1 mutants independent of the observed SOD1 blockade in nonendothelial cells and/or endothelia.

A convergence of evidence has led to a consensus that SOD1 mutations cause disease by acquisition of 1 or more toxic properties, rather than by loss of dismutase activity, including mutant damage to mitochondria, damage from aberrant mutant SOD1 secretion (45), endoplasmic reticulum stress from blockage of ejection of misfolded proteins from it (46), and hyperactivation of extracellular superoxide production by microglia (47), as reviewed recently (48). We would emphasize that whatever the most relevant toxicities, APC-mediated mutant SOD1 downregulation within motor neurons and microglia represents a therapeutic approach that is directly linked to disease mechanism. APC-mediated diminution of mutant SOD1 synthesis in motor neurons may contribute to the delay in disease initiation, whereas - perhaps more importantly - lowered SOD1 mutant levels within astrocytes, microglia, or peripheral macrophages are highly likely to be responsible for slowed disease progression. This conclusion would be in accordance with prior findings that selective mutant SOD1 gene excision from astrocytes (17), from microglia and peripheral macrophage lineages $(16,49)$, or by bone marrow replacement of mutant myeloid cells with normal ones (50) can strikingly slow disease progression despite no effect on disease onset.

The only other SOD1 gene-silencing approach previously proven to slow disease progression is antisense DNA oligonucleotide infusion (51), but these oligonucleotides are not BBB or BSCB permeant, requiring delivery by direct $\mathrm{CNS}$ infusion following invasive surgery. Methods of gene silencing through retroviral delivery of transcription-mediated shRNAs have been shown to dramatically slow disease onset, but only when administered to very young SOD1 ${ }^{\mathrm{G} 93 \mathrm{~A}}$ animals. Even with delivery at a juvenile age, this approach was of no benefit in slowing the rate of disease progression (52). Modest survival benefits from slowing disease onset, but without benefit in slowing progression, have also been seen with viral delivery prior to disease onset by direct injection into the spinal cord to very focally silence SOD1 (53). Thus, the ability of APC to slow disease progression, combined with simple peripheral administration, represent unique therapeutic advantages compared with other SOD1-silencing strategies. Moreover, APC is already approved for use in adult patients. Indications include severe sepsis (54), and a clinical trial is currently underway to assess the benefit of APC in acute ischemic stroke (http://www. clinicaltrials.gov/ct2/show/NCT00533546). What is clear from our efforts and the prior ones $(5,6,13,14,55)$ is that transient exposure, without continuous infusion, of APC can produce longlasting neuroprotective effects. With the recognition that accumulation of aberrant SOD1 species has been linked to most cases of sporadic ALS (56), strategies based on activation of the protein C cellular pathway are promising directions for treating patients with familial, and possibly sporadic, ALS.

\section{Methods}

Mouse recombinant APC variants. WT-APC, S360A-APC, 3K3A-APC, and $5 \mathrm{~A}-\mathrm{APC}$ were prepared as described previously $(5,21)$.

APC treatment of SOD $1^{\mathrm{G} 93 A}$ mice. Studies were performed in male SOD $1^{\mathrm{G} 93 \mathrm{~A}}$ mice (The Jackson Laboratory) according to NIH guidelines, using a protocol approved by the University of Rochester. Mice were injected i.p. daily with saline or APC analogs after disease onset and continued throughout the symptomatic stage until death. Disease onset was determined from the weight curves $(16,25-27)$. Mice were randomized into 5 groups receiving saline ( $n=19)$, WT-APC $(40 \mu \mathrm{g} / \mathrm{kg} / \mathrm{d} ; n=10), 3 \mathrm{~K} 3 \mathrm{~A}-\mathrm{APC}(40 \mu \mathrm{g} / \mathrm{kg} / \mathrm{d} ; n=11)$, S360A-APC $(100 \mu \mathrm{g} / \mathrm{kg} / \mathrm{d} ; n=10)$, and 5A-APC $(100 \mu \mathrm{g} / \mathrm{kg} / \mathrm{d} ; n=10)$. Mortality was defined when the mouse could not right itself within 30 seconds.

APC arterial plasma profiles. Mice ( $n=3$ per group) were anesthetized i.p. with $100 \mathrm{mg} / \mathrm{kg}$ ketamine and $10 \mathrm{mg} / \mathrm{kg}$ xylazine. WT-APC (40 or $100 \mu \mathrm{g} /$ $\mathrm{kg})$ and 5A-APC $(100 \mu \mathrm{g} / \mathrm{kg})$ were administered i.p., and blood samples were collected from the femoral artery.

Amyloidotic assay, coagulation assay, and APC ELISA. Assays were performed as reported previously $(30,57)$. See Supplemental Methods.

${ }^{125}$ I-5A-APC uptake into the lumbar spinal cord. Studies were performed according to the $\mathrm{NIH}$ guidelines using a protocol approved by the University of Rochester. 5A-APC was radioiodinated as reported previously (29). Male C57BL/ 6 mice, severely depleted EPCR mice (58), PAR1-null mice, SOD1 1933A mice, and nontransgenic littermate controls weighing about 23-24 g were anesthetized i.p. with ketamine $(100 \mathrm{mg} / \mathrm{kg})$ and xylazine $(10 \mathrm{mg} / \mathrm{kg})$ and injected with ${ }^{125} \mathrm{I}-5 \mathrm{~A}-\mathrm{APC}$ (MW, $56 \mathrm{kDa} ; 100 \mu \mathrm{g} / \mathrm{kg}$ i.p. or $1.25 \mu \mathrm{Ci}$ per mouse) simultaneously with ${ }^{99 \mathrm{~m}} \mathrm{Tc}$-albumin (MW, $67 \mathrm{kDa} ; 10 \mu \mathrm{Ci}$, Cardinal Health), a reference vascular space marker (59). Plasma samples were collected from the femoral artery. Mice were sacrificed, and the lumbar region was carefully dissected and prepared for counting. See Supplemental Methods for details regarding iodination, counting, and calculations $(19,59)$.

Treatment of EPCR $/ \delta$ bypomorphs with 5A-APC. Severely depleted male EPCR mice (58) and their littermate controls (2-3 months old) were treated with $100 \mu \mathrm{g} / \mathrm{kg} / \mathrm{d} 5 \mathrm{~A}-\mathrm{APC}$ i.p. or saline for 7 days. Animals were sacrificed at day 7. SOD1 mRNA levels were determined in laser-captured motor neurons and microvessels by QPCR analysis. SOD1 protein levels were analyzed by immunoblotting of isolated motor neurons and spinal cord microvessels lysates (see below). Blockade of EPCR in SOD1 ${ }^{\mathrm{G} 93 \mathrm{~A}}$ mice is described in Supplemental Methods.

Laser capture microdissection. The lumbar (L2-L5) OCT-embedded 10- $\mu \mathrm{m}$ tick spinal cord sections were mounted on RNase-free PALM membrane slides (Carl Zeiss Microimaging). Motor neurons were stained using cresyl violet (26). Microvessels were stained with a rabbit polyclonal anti-mouse laminin antibody (diluted 1:500; Sigma-Aldrich). HRP-conjugated goat anti-rabbit antibody was used as a secondary antibody (diluted 1:200; 
Dako North America). Microglia were stained with a rat monoclonal antimouse CD11b (diluted 1:100; BD Biosciences - Pharmingen). Contamination-free laser capture microdissection was performed on dry, stained sections at $\times 400$ magnification with a Zeiss Axiovert 200 inverted microscope equipped with PALM LCM system including a 337-nm laser and a robotic microscope table operated by PalmRobo software. Motor neurons, microvessels, and microglia were captured from 10-12 randomly chosen sections of the ventral horns of SOD $1^{\mathrm{G} 93 \mathrm{~A}}$ mice treated with saline, S360AAPC, $5 \mathrm{~A}-\mathrm{APC}$, or WT-APC ( $n=5$ per group).

Motor neuron-enriched cell suspension. Motor neurons were isolated as reported previously (60). See Supplemental Methods.

Isolation of microvessels by dextran density centrifugation gradient. The spinal cord microvessels were isolated as described previously (19). See Supplemental Methods.

N2a cultures. N2a-SOD1 ${ }^{\mathrm{WT}}$, N2a-SOD1 $1^{\mathrm{G} 37 \mathrm{R}}$, and N2a-SOD $1^{\mathrm{G} 85 \mathrm{R}}$ cells were cultured as described previously (61).

Oxidant stress. Cells were challenged with $10 \mu \mathrm{M}$ mouse $\mathrm{Hb}$ (19) or with xanthine/xanthine oxidase $(100 \mu \mathrm{M}$ to $10 \mathrm{mU} / \mathrm{ml}$; refs. 42,61$)$ in the presence and absence of $5 \mathrm{nM} 5 \mathrm{~A}-\mathrm{APC}$ or S360A-APC. Cell viability was determined with a water-soluble tetrazolium-8 assay kit (CCK-8 kit; Dojindo Molecular Technologies).

NMDA-induced apoptosis in N2a-SOD1 ${ }^{G 85 R}$ cells. This was performed as described previously $(7,30)$. See Supplemental Methods.

Inhibition of PARs. Antibodies against PARs were from Santa Cruz Biotechnology Inc. The following cleavage site-blocking PAR antibodies were used, each highly specific for blocking its respective PAR: polyclonal rabbit against human PAR1 (H-111), monoclonal mouse against human PAR2 (SAM-11), polyclonal rabbit against human PAR3 (H-103), and polyclonal goat against mouse PAR4 (S-20). See Supplemental Methods.

Silencing through RNA interference. See Supplemental Methods.

Immunostaining and immunoblot analysis for Sp1. See Supplemental Methods. Immunoblot analysis. Microvessels, motor neurons, and N2a cell lysates were prepared in the Cell Lysis Buffer (Cell Signaling Technology). We analyzed 20-40 $\mu \mathrm{g}$ lysate proteins using $10 \%$ SDS-PAGE. Reactivity was detected using an enhanced chemiluminescence detection system (Amersham). The density of bands was quantified by scanning densitometry relative to $\beta$-actin signal (Alpha Imager; Alpha Innotech). For each studied protein, the signal was within the linear range. See Supplemental Methods for antibodies.

Real-time QPCR. Total RNA was isolated from laser-captured motor neurons, microvessels and microglia, and N2a cells using RNeasy Mini kit (Qiagen Inc.) and reverse transcripted to cDNA using the iScript cDNA Synthesis kit (Bio-Rad Laboratories). The cDNA products of the RT reaction were stored at $-80^{\circ} \mathrm{C}$ or used immediately for QPCR. QPCR, using iQ SYBR Green Supermix (Bio-Rad Laboratories) as the fluorescent DNA intercalating agent, was analyzed using a IQ4 multicolor detection QPCR system (Bio-Rad Laboratories). The relative abundance of target mRNA was normalized to $\beta$-actin. See Supplemental Methods for primers.

Endothelial-specific mutant SOD $1^{\mathrm{G} 37 R}$ deletion. The deletion of mutant SOD $1^{\mathrm{G} 37 \mathrm{R}}$ gene from endothelial cells was accomplished using Ve-cadherin-Cre mice (39) with selective expression of the Cre recombinase in endothelial cells and a transgenic line carrying SOD1G37R mutant (16). This approach has been successfully used to delete SOD1 mutant gene in microglia (16), motor neurons $(16,17)$, and astrocytes (17). The gene excision efficiency was determined by QPCR of SOD1G37R DNA in microvessels from brain and spinal cord (see below). See Supplemental Methods for measurements of BSCB disruption.
DNA extraction from microvessels in SOD1 $1^{G 37 R}$ mice and QPCR. Vessels from brain and spinal cords were isolated as described above. DNA was extracted as described previously (62). For QPCR, 30 ng DNA was amplified as described previously (63). SOD1 and apoB primer/probe sets were run in the same reaction, with every reaction run in triplicates and all samples run in parallel. The entire experiment was repeated twice, and results were averaged.

Histology and immunohistochemistry in SOD $1{ }^{\mathrm{G} 93 \mathrm{~A}}$ mice. Mice were anesthetized i.p. with $100 \mathrm{mg} / \mathrm{kg}$ ketamine and $10 \mathrm{mg} / \mathrm{kg}$ xylazine and perfused transcardially with heparinized PBS. Lumbar spinal cords (L2-L5 region) were embedded in OCT medium (Sakura) and cut in the coronal plane at $14 \mu \mathrm{m}$.

Serum protein leakage. Sections were fixed in acetone for 5 minutes, blocked by $5 \%$ swine serum for 1 hour at room temperature, and incubated with fluorescein-conjugated affinity-purified goat anti-mouse IgG (fluorescein-labeled primary antibody, diluted 1:200; Jackson ImmunoResearch Laboratories) and rat monoclonal anti-mouse CD31 (endothelial marker, diluted 1:100; BD Biosciences - Pharmingen). Alexa Fluor 594 donkey anti-rat IgG (diluted 1:200; Invitrogen) was used as a secondary antibody to CD31. Images were taken using a Zeiss 510 meta confocal microscope. In each mouse, 12 nonadjacent sections (greater than $250 \mu \mathrm{m}$ apart in the L2-L5 region) were examined. The signal intensity of mouse IgG extravascular deposits was analyzed using NIH Image J. Sections of all control and experimental mice were processed in parallel.

Prussian blue staining. Staining was performed as described previously (19). See Supplemental Methods.

Immunofluorescent staining of microglia. We used rat monoclonal antimouse CD11b (diluted 1:100; BD Biosciences - Pharmingen), and nuclei were stained with DAPI. Secondary antibody was Alexa Fluor 594 donkey anti-rat IgG (diluted 1:200; Invitrogen). All sections from control and experimental animals were processed in parallel.

Statistics. We used the Cox proportional hazards model (64), with the treatment group as the unique covariate, to study the effect of treatment on the survival distributions and lifespan of mice from different treatment groups. Symptomatic phase duration was calculated in days as the difference between lifespan and disease onset. We used 1-way ANOVA followed by Tukey post-hoc test to calculate differences in symptomatic phase as well as to compare the treatment effects of ordinal data between groups. S-plus 7.0 was used for statistical calculations. All data are mean \pm SEM unless otherwise indicated. A $P$ value less than 0.05 was considered significant.

\section{Acknowledgments}

This work was supported by NIH grants HL63290 and HL81528 (to B.V. Zlokovic), HL73750 (to F.J. Castellino), HL52246 (to J.H. Griffin), and NS27036 (to D.W. Cleveland), as well as by Socratech LLC. D.W. Cleveland receives salary support from the Ludwig Institute for Cancer Research. H. I receives salary support from a Career Development Award from the Muscular Dystrophy Association. The authors thank Xin Tu for help with statistical analysis.

Received for publication January 5, 2009, and accepted in revised form September 9, 2009.

Address correspondence to: Berislav V. Zlokovic, Arthur Kornberg Medical Research Building, University of Rochester Medical Center, 601 Elmwood Avenue, Box 645, Rochester, New York 14642, USA. Phone: (585) 273-3132; Fax: (585) 273-3133; E-mail: berislav_zlokovic@urmc.rochester.edu.
1. Mosnier, L.O., Zlokovic, B.V., and Griffin, J.H. 2007. The cytoprotective protein C pathway. Blood. 109:3161-3172.

2. Joyce, D.E., Gelbert, L., Ciaccia, A., DeHoff, B., and
Grinnell, B.W. 2001. Gene expression profile of antithrombotic protein $\mathrm{c}$ defines new mechanisms modulating inflammation and apoptosis. J. Biol. Chem. 276:11199-11203.
3. Riewald, M., and Ruf, W. 2005. Protease-activated receptor-1 signaling by activated protein $\mathrm{C}$ in cytokine-perturbed endothelial cells is distinct from thrombin signaling. J. Biol. Chem. 280:19808-19814. 
4. Riewald, M., Petrovan, R.J., Donner, A., Mueller, B.M., and Ruf, W. 2002. Activation of endothelial cell protease activated receptor 1 by the protein C pathway. Science. 296:1880-1882.

5. Cheng, T., et al. 2003. Activated protein C blocks p53-mediated apoptosis in ischemic human brain endothelium and is neuroprotective. Nat. Med. 9:338-342.

6. Cheng, T., et al. 2006. Activated protein C inhibits tissue plasminogen activator-induced brain hemorrhage. Nat. Med. 12:1278-1285.

7. Guo, H., et al. 2004. Activated protein C prevents neuronal apoptosis via protease activated receptors 1 and 3. Neuron. 41:563-572.

8. Feistritzer, C., and Riewald, M. 2005. Endothelial barrier protection by activated protein $C$ through PAR1-dependent sphingosine 1-phosphate receptor-1 crossactivation. Blood. 105:3178-3184.

9. Finigan, J.H., et al. 2005. Activated protein C mediates novel lung endothelial barrier enhancement: role of sphingosine 1-phosphate receptor transactivation. J. Biol. Chem. 280:17286-17293.

10. Isermann, B., et al. 2007. Activated protein C protects against diabetic nephropathy by inhibiting endothelial and podocyte apoptosis. Nat. Med. 13:1349-1358.

11. Liu, D., et al. 2004. Tissue plasminogen activator neurovascular toxicity is controlled by activated protein C. Nat. Med. 10:1379-1383.

12. Zlokovic, B.V., et al. 2005. Functional recovery after embolic stroke in rodents by activated protein C. Ann. Neurol. 58:474-477.

13. Thiyagarajan, M., Fernandez, J.A., Lane, S.M., Griffin, J.H., and Zlokovic, B.V. 2008. Activated protein $\mathrm{C}$ promotes neovascularization and neurogenesis in postischemic brain via protease-activated receptor 1. J. Neurosci. 28:12788-12797.

14. Han, M.H., et al. 2008. Proteomic analysis of active multiple sclerosis lesions reveals therapeutic targets. Nature. 451:1076-1081.

15. Boillee, S., Vande, V.C., and Cleveland, D.W. 2006. ALS: a disease of motor neurons and their nonneuronal neighbors. Neuron. 52:39-59.

16. Boillee, S., et al. 2006. Onset and progression in inherited ALS determined by motor neurons and microglia. Science. 312:1389-1392.

17. Yamanaka, K., et al. 2008. Astrocytes as determinants of disease progression in inherited amyotrophic lateral sclerosis. Nat. Neurosci. 11:251-253.

18. Garbuzova-Davis, S., et al. 2007. Evidence of compromised blood-spinal cord barrier in early and late symptomatic SOD1 mice modeling ALS. PLoS. ONE. 2:e1205

19. Zhong, Z., et al. 2008. ALS-causing SOD1 mutants generate vascular changes prior to motor neuron degeneration. Nat. Neurosci. 11:420-422.

20. Mosnier, L.O., Gale, A.J., Yegneswaran, S., and Griffin, J.H. 2004. Activated protein $C$ variants with normal cytoprotective but reduced anticoagulant activity. Blood. 104:1740-1744.

21. Mosnier, L.O., Yang, X.V., and Griffin, J.H. 2007. Activated protein $\mathrm{C}$ mutant with minimal anticoagulant activity, normal cytoprotective activity, and preservation of thrombin activable fibrinolysis inhibitor-dependent cytoprotective functions. J. Biol. Chem. 282:33022-33033.

22. Kerschen, E.J., et al. 2007. Endotoxemia and sepsis mortality reduction by non-anticoagulant activated protein C. J. Exp. Med. 204:2439-2448.

23. Gurney, M.E., et al. 1994. Motor neuron degeneration in mice that express a human $\mathrm{Cu}, \mathrm{Zn}$ superoxide dismutase mutation. Science. 264:1772-1775.

24. Gale, A.J., Tsavaler, A., and Griffin, J.H. 2002. Molecular characterization of an extended binding site for coagulation factor $\mathrm{Va}$ in the positive exosite of activated protein C. J. Biol. Chem. 277:28836-28840

25. Schutz, B., et al. 2005. The oral antidiabetic pio- glitazone protects from neurodegeneration and amyotrophic lateral sclerosis-like symptoms in superoxide dismutase-G93A transgenic mice. J. Neurosci. 25:7805-7812.

26. Lobsiger, C.S., Boillee, S., and Cleveland, D.W 2007. Toxicity from different SOD1 mutants dysregulates the complement system and the neuronal regenerative response in ALS motor neurons. Proc. Natl. Acad. Sci. U. S. A. 104:7319-7326.

27. Kieran, D., et al. 2004. Treatment with arimoclomol, a coinducer of heat shock proteins, delays disease progression in ALS mice. Nat. Med. 10:402-405.

28. Gale, A.J., Sun, X., Heeb, M.J., and Griffin, J.H. 1997. Nonenzymatic anticoagulant activity of the mutant serine protease Ser360Ala-activated protein C mediated by factor Va. Protein Sci. 6:132-140.

29. Deane, R., et al. 2009. Endothelial protein C receptor-assisted transport of activated protein $\mathrm{C}$ across the mouse blood-brain barrier. J. Cereb. Blood Flow Metab. 29:25-33.

30. Guo, H., et al. 2009. Species-dependent neuroprotection by activated protein $\mathrm{C}$ mutants with reduced anticoagulant activity. J. Neurochem. 109:116-124.

31. McLaughlin, J.N., Patterson, M.M., and Malik, A.B. 2007. Protease-activated receptor-3 (PAR3) regulates PAR1 signaling by receptor dimerization. Proc. Natl. Acad. Sci. U. S. A. 104:5662-5667.

32. Esmon, C.T. 2000. The endothelial cell protein C receptor. Thromb. Haemost. 83:639-643.

33. Thiyagarajan, M., Cheng, T., and Zlokovic, B.V. 2007. Endothelial cell protein $C$ receptor: role beyond endothelium? Circ. Res. 100:155-157.

34. Gorbacheva, L.R., et al. 2008. Activated protein C via PAR1 receptor regulates survival of neurons under conditions of glutamate excitotoxicity. Biochemistry (Mosc.). 73:717-724.

35. Minc, E., et al. 1999. The human copper-zinc superoxide dismutase gene (SOD1) proximal promoter is regulated by Sp1, Egr-1, and WT1 via non-canonical binding sites. J. Biol. Chem. 274:503-509.

36. Chang, M.S., Yoo, H.Y., and Rho, H.M. 1999. Positive and negative regulatory elements in the upstream region of the rat $\mathrm{Cu} / \mathrm{Zn}$-superoxide dismutase gene. Biochem. J. 339:335-341.

37. Weintraub, H., et al. 1991. The myoD gene family: nodal point during specification of the muscle cell lineage. Science. 251:761-766.

38. Scholz, M., et al. 2004. Thrombin induces Sp1mediated antiviral effects in cytomegalovirusinfected human retinal pigment epithelial cells. Med. Microbiol. Immunol. 193:195-203.

39. Alva, J.A., et al. 2006. VE-Cadherin-Cre-recombinase transgenic mouse: a tool for lineage analysis and gene deletion in endothelial cells. Dev. Dyn. 235:759-767.

40. Henkel, J.S., Beers, D.R., Siklos, L., and Appel, S.H. 2006. The chemokine MCP-1 and the dendritic and myeloid cells it attracts are increased in the MSOD 1 mouse model of ALS. Mol. Cell Neurosci. 31:427-437.

41. Ye, X., et al. 1999. The endothelial cell protein C receptor (EPCR) functions as a primary receptor for protein $\mathrm{C}$ activation on endothelial cells in arteries, veins, and capillaries. Biochem. Biophys. Res. Commun. 259:671-677

42. Lambrechts, D., et al. 2003. VEGF is a modifier of amyotrophic lateral sclerosis in mice and humans and protects motoneurons against ischemic death. Nat. Genet. 34:383-394.

43. Oosthuyse, B., et al. 2001. Deletion of the hypoxia-response element in the vascular endothelial growth factor promoter causes motor neuron degeneration. Nat. Genet. 28:131-138.

44. Niessen, F., et al. 2009. Endogenous EPCR/aPCPAR1 signaling prevents inflammation-induced vascular leakage and lethality. Blood. 113:2859-2866.

45. Urushitani, M., et al. 2006. Chromogranin-mediated secretion of mutant superoxide dismutase proteins linked to amyotrophic lateral sclerosis. Nat. Neurosci. 9:108-118.

46. Saxena, S., Cabuy, E., and Caroni, P. 2009. A role for motoneuron subtype-selective ER stress in disease manifestations of FALS mice. Nat. Neurosci. 12:627-636.

47. Harraz, M.M., et al. 2008. SOD1 mutations disrupt redox-sensitive Rac regulation of NADPH oxidase in a familial ALS model. J. Clin. Invest. 118:659-670.

48. Polymenidou, M., and Cleveland, D.W. 2008. Motor neuron disease: The curious ways of ALS. Nature. 454:284-285.

49. Wang, L., Sharma, K., Grisotti, G., and Roos, R.P. 2009. The effect of mutant SOD1 dismutase activity on non-cell autonomous degeneration in familial amyotrophic lateral sclerosis. Neurobiol. Dis. 35:234-240.

50. Beers, D.R., et al. 2006. Wild-type microglia extend survival in PU.1 knockout mice with familial amyotrophic lateral sclerosis. Proc. Natl. Acad. Sci. U. S. A. 103:16021-16026.

51. Smith, R.A., et al. 2006. Antisense oligonucleotide therapy for neurodegenerative disease. J. Clin. Invest. 116:2290-2296

52. Raoul, C., et al. 2005. Lentiviral-mediated silencing of SOD1 through RNA interference retards disease onset and progression in a mouse model of ALS. Nat. Med. 11:423-428.

53. Ralph, G.S., et al. 2005. Silencing mutant SOD1 using RNAi protects against neurodegeneration and extends survival in an ALS model. Nat. Med. 11:429-433.

54. Bernard, G.R., et al. 2001. Efficacy and safety of recombinant human activated protein $\mathrm{C}$ for severe sepsis. N. Engl. J. Med. 344:699-709.

55. Domotor, E., et al. 2003. Activated protein C alters cytosolic calcium flux in human brain endothelium via binding to endothelial protein $C$ receptor and activation of protease activated receptor-1. Blood. 101:4797-4801

56. Gruzman, A., et al. 2007. Common molecular signature in SOD1 for both sporadic and familial amyotrophic lateral sclerosis. Proc. Natl. Acad. Sci. U. S. A. 104:12524-12529.

57. Fernandez, J.A., Lentz, S.R., Dwyre, D.M., and Griffin, J.H. 2006. A novel ELISA for mouse activated protein C in plasma. J. Immunol. Methods. 314:174-181.

58. Castellino, F.J., et al. 2002. Mice with a severe deficiency of the endothelial protein $\mathrm{C}$ receptor gene develop, survive, and reproduce normally, and do not present with enhanced arterial thrombosis after challenge. Thromb. Haemost. 88:462-472.

59. Deane, R., et al. 2005. IgG-assisted age-dependent clearance of Alzheimer's amyloid beta peptide by the blood-brain barrier neonatal Fc receptor. J. Neurosci. 25:11495-11503.

60. Liu, Z., and Martin, L.J. 2001. Isolation of mature spinal motor neurons and single-cell analysis using the comet assay of early low-level DNA damage induced in vitro and in vivo. J. Histochem. Cytochem. 49:957-972.

61. Pasinelli, P., Houseweart, M.K., Brown, R.H., Jr., and Cleveland, D.W. 2000. Caspase- 1 and -3 are sequentially activated in motor neuron death in $\mathrm{Cu}, \mathrm{Zn}$ superoxide dismutase-mediated familial amyotrophic lateral sclerosis. Proc. Natl. Acad. Sci. U. S. A. 97:13901-13906.

62. Legros, F., Malka, F., Frachon, P., Lombes, A., and Rojo, M. 2004. Organization and dynamics of human mitochondrial DNA. J. Cell Sci. 117:2653-2662.

63. Lobsiger, C.S., et al. 2009. Schwann cells expressing dismutase active mutant SOD1 unexpectedly slow disease progression in ALS mice. Proc. Natl. Acad. Sci. U. S. A. 106:4465-4470.

64. Cox, D.R. 1972. Regression models and life tables (with discussion). J. Royal Stat. Soc. Series B. 34:187-220. 